\title{
REGULARITY OF ALGEBRAS RELATED TO THE SKLYANIN ALGEBRA
}

\author{
J. T. STAFFORD
}

\begin{abstract}
This paper continues the research of [SS] by finding further examples of (Artin-Schelter) regular rings of dimension four. Unlike the threedimensional case studied in [ATV1, 2], these examples show that the fourdimensional regular rings are not uniquely determined by their associated geometric data. Indeed, we find a one-parameter family of regular algebras associated to this data.
\end{abstract}

\section{INTRODUCTION}

0.1 . Fix once and for all an algebraically closed field $k$ of characteristic not equal to 2. Throughout this paper, a graded algebra $A$ will mean a (connected) $\mathbb{N}$-graded algebra, generated in degree 1; thus $A=\bigoplus_{i \geq 0} A_{i}$, where $A_{0}=k$ is central, $\operatorname{dim}_{k} A_{i}<\infty$ for all $i$, and $A$ is generated as an algebra by $A_{1}$. Following [AS], a graded ring $A$ is called regular (of dimension $d$ ) if

(i) $A$ has finite global homological dimension, gldim $A=d$;

(ii) $A$ has finite Gelfand-Kirillov dimension, GKdim $A<\infty$; that is, there exists $\rho \in \mathbb{R}$ such that $\operatorname{dim} A_{n} \leq n^{\rho}$ for all $n$.

(iii) $A$ is Gorenstein; that is, $\operatorname{Ext}_{A}^{q}(k, A)=\delta_{d, q} \cdot k$.

0.2. The papers [ATV1, 2] classify all regular graded rings of dimension 3 and, in doing so, introduce some novel geometric techniques into the study of noncommutative ring theory. Those techniques were shown to have an analogue in dimension 4 in [SS] and this paper continues the research of [SS].

To describe these techniques and the questions that motivated this paper, assume that $A$ is either one of the nontrivial examples considered in [ATV1] (specifically, assume that $A$ is one of the three-generated, type A examples of [AS]), or that $A$ is a Sklyanin algebra, as considered in [SS] and defined in $(0.3)$ below. The important properties of either ring $A$ is that it is a graded algebra with $n=3$ (respectively $n=4$ ) generators, defined by $\frac{1}{2} n(n-1)$ quadratic relations. Moreover, $A$ cannot be studied by the more traditional methods of noncommutative ring theory: in particular, it is not an iterated Ore extension and does not have any natural PBW basis. The basic idea of [ATV] and [SS] is that one can associate to the ring $A$ an elliptic curve $E \subset \mathbb{P}^{n}=\mathbb{P}_{k}^{n}$ and an automorphism $\tau$ of $E$. Corresponding to this data there exists a natural "twisted

Received by the editors June 30, 1991 and, in revised form, January 30, 1992.

1991 Mathematics Subject Classification. Primary 16A33, 16A58, 16A60, 14 H52.

Key words and phrases. Noncommutative Noetherian ring, regular ring, elliptic curve.

This research was supported in part by an NSF grant. 
homogeneous coordinate ring" $B$ of $E$ that is automatically a homomorphic image of $A$. The advantage of this construction is that the geometry of $E$ can be used to obtain very detailed information about $B$. This is in turn used to study the structure of $A$; proving, for example, that $A$ is a regular, Noetherian domain with the with the same Hilbert series as a (commutative) polynomial ring in $n$ variables.

A number of questions remain about regular rings, even in dimensions 3 and 4, and about the geometric techniques introduced by [ATV]. In particular, the way in which the given information about $B$ is used to study the $\operatorname{ring} A$ involves a complicated induction that gives rather little information as to why the proof should work. Moreover, this proof seems to require surprisingly little information about $A$ other than it map onto $B$ and be a quadratic algebra; that is it is a graded algebra defined by quadratic relations. In dimension 3 , this is somewhat obscured by the fact that $B$ is defined by the quadratic defining relations of $A$, together with one cubic relation. Thus $A$ is uniquely determined by $B$. However, in dimension $4 B$ is also a quadratic algebra, as it is obtained by factoring out two quadratic elements from $A$. Thus, there is no obvious way in which $A$ is determined by $B$. In the introduction to [ATV1], the authors isolate this as one of the significant problems in any attempt to generalize their results to higher dimensions.

This leads to the questions that motivated the present research: Given the geometric ring $B$ obtained from the Sklyanin algebra $A$, to what extent is $A$ determined by $B$ ? Alternatively, what is required of a quadratic algebra $A^{\prime}$, other than it have four generators, six relations, and map onto $B$, that are needed for it to be regular. Surprisingly, and as was suggested above, one really does need to demand relatively little of $A^{\prime}$. Indeed, the main result of this paper shows that for each of these rings $B$ there is a one-parameter family of regular rings $A^{\prime}$ that map onto $B$, and each of these rings satisfies the same basic properties as the Sklyanin algebra $A$. While this suggests that there is little hope of classifying the regular graded rings of dimension $d \geq 4$, it does say that there ought to be a large number of rings for which the techniques of [ATV] are applicable.

0.3. We have delayed giving an explicit description of the main results of this paper for too long. Fix scalars $\alpha_{1}, \alpha_{2}, \alpha_{3} \in k$ that satisfy

$$
\alpha_{1}+\alpha_{2}+\alpha_{3}+\alpha_{1} \alpha_{2} \alpha_{3}=0 \text {. }
$$

The Sklyanin algebra $S=S\left(\alpha_{1}, \alpha_{2}, \alpha_{3}\right)$ is the graded $k$-algebra with generators $x_{0}, x_{1}, x_{2}, x_{3}$ of degree 1 , and relations $f_{i}=0$, where

$$
\begin{aligned}
& f_{1}=x_{0} x_{1}-x_{1} x_{0}-\alpha_{1}\left(x_{2} x_{3}+x_{3} x_{2}\right), \\
& f_{2}=x_{0} x_{1}+x_{1} x_{0}-\left(x_{2} x_{3}-x_{3} x_{2}\right), \\
& f_{3}=x_{0} x_{2}-x_{2} x_{0}-\alpha_{2}\left(x_{3} x_{1}+x_{1} x_{3}\right), \\
& f_{4}=x_{0} x_{2}+x_{2} x_{0}-\left(x_{3} x_{1}-x_{1} x_{3}\right), \\
& f_{5}=x_{0} x_{3}-x_{3} x_{0}-\alpha_{3}\left(x_{1} x_{2}+x_{2} x_{1}\right), \\
& f_{6}=x_{0} x_{3}+x_{3} x_{0}-\left(x_{1} x_{2}-x_{2} x_{1}\right) .
\end{aligned}
$$

This algebra was introduced in [Sk1, 2] in connection with Sklyanin's work on the "quantum inverse scattering method". However, our interest in this algebra stems from: 
Theorem [SS, Theorem 0.3]. Assume that

$$
\left\{\alpha_{1}, \alpha_{2}, \alpha_{3}\right\} \cap\{0, \pm 1\}=\varnothing .
$$

Then $S$ is a Noetherian domain, with the same Hilbert series

$$
H_{S}(t)=\sum \operatorname{dim}_{k}\left(S_{n}\right) t^{n}=1 /(1-t)^{4}
$$

as that of a commutative polynomial ring in four variables. Moreover, $S$ is a regular graded algebra of dimension 4 and is a Koszul algebra; that is, the Yoneda Ext algebra $\operatorname{Ext}_{S}^{*}(k, k)=\bigoplus \operatorname{Ext}_{S}^{n}(k, k)$ is generated by $\operatorname{Ext}^{1}(k, k)$.

0.4. Set

(0.4.1) $\Omega_{1}=-x_{0}^{2}+x_{1}^{2}+x_{2}^{2}+x_{3}^{2} \quad$ and $\quad \Omega_{2}=x_{1}^{2}+\frac{1+\alpha_{1}}{1-\alpha_{2}} x_{2}^{2}+\frac{1-\alpha_{1}}{1+\alpha_{3}} x_{3}^{2}$.

Then the $\Omega_{i}$ are central in $S$ and the geometric ring $B$ is the factor ring $S / \Omega_{1} S+\Omega_{2} S$. The aim of this paper is to find other quadratic algebras, with four generators and six relations, that have $B$ as a homomorphic image and satisfy the conclusions of Theorem 0.3 . The rings for which we can prove this are defined as follows. Given $d=\left(d_{1}, d_{2}\right) \in \mathbb{P}_{k}^{1}$ and $1 \leq i \leq 6$, set

$$
\begin{aligned}
S_{d, i} & =S_{d, i}\left(\alpha_{1}, \alpha_{2}, \alpha_{3}\right) \\
& =k\left\{x_{0}, x_{1}, x_{2}, x_{3}\right\} /\left(d_{1} \Omega_{1}+d_{2} \Omega_{2}, \text { and } f_{j} ; 1 \leq j \leq 6, j \neq i\right) .
\end{aligned}
$$

(Since the $\Omega_{j}$ are both homogeneous of the same degree, this is independent of the homogeneous coordinates of $d \in \mathbb{P}^{1}$.) Similarly, let

$$
S_{\infty}=S_{\infty}\left(\alpha_{1}, \alpha_{2}, \alpha_{3}\right)=k\left\{x_{0}, x_{1}, x_{2}, x_{3}\right\} /\left(f_{1}, f_{2}, f_{3}, f_{4}, \Omega_{1}, \Omega_{2}\right) .
$$

Then we prove:

Theorem. Assume the $\alpha_{i}$ satisfy (0.3.1) and (0.3.3) and let $R=S_{d, j}\left(\alpha_{1}, \alpha_{2}, \alpha_{3}\right)$ for some $d=\left(d_{1}, d_{2}\right) \in \mathbb{P}_{k}^{1}$ and $1 \leq j \leq 2$ or let $R=S_{\infty}\left(\alpha_{1}, \alpha_{2}, \alpha_{3}\right)$. If $R=S_{d, 1}$, assume that $d \neq(1,0),\left(1,-1-\alpha_{2} \alpha_{3}\right)$ and if $R=S_{d, 2}$ assume that $d \neq\left(1, \alpha_{2}-1\right),\left(1,-1-\alpha_{3}\right)$. Then the following statements hold.

(i) $R$ is an (Artin-Schelter) regular graded algebra of dimension 4 .

(ii) $H_{R}(t)=1 /(1-t)^{4}$.

(iii) $R$ is a Noetherian domain.

(iv) $R$ is a Koszul algebra.

Finally, let $\left\{\alpha_{i}\right\},\left\{\beta_{i}\right\}$, and $\left\{\gamma_{i}\right\}$ satisfy (0.3.1) and (0.3.3). Then there exists no isomorphism between the three rings $S\left(\alpha_{1}, \alpha_{2}, \alpha_{3}\right), S_{d, i}\left(\beta_{1}, \beta_{2}, \beta_{3}\right)$, and $S_{\infty}\left(\gamma_{1}, \gamma_{2}, \gamma_{3}\right)$.

One could also give a version of the theorem for the rings $S_{d, j}$ for $3 \leq j \leq 6$, and the analogues of $S_{\infty}$ obtained by omitting $f_{3}, f_{4}$ or $f_{5}, f_{6}$ from the relations. However, cyclically permuting the variables $\left\{x_{1}, x_{2}, x_{3}\right\}$ provides an isomorphism from each of these new rings to one of the ones described in the statement of the theorem.

We remark that, just as the Sklyanin algebra can be regarded as an elliptic deformation of the polynomial ring in four variables, so the ring $S_{d, 1}$ can be regarded as an elliptic deformation of the ring

$A=k\left\{x_{0}, x_{1}, x_{2}, x_{3}\right\} /\left(x_{0}^{2}+x_{1}^{2}+x_{2}^{2}+x_{3}^{2}\right.$, and $\left.x_{i} x_{j}-x_{j} x_{i}:(i, j) \neq(2,3)\right)$

It is not clear whether there is anything particularly distinguished about this ring $A$. 
0.5. Combined with results from [Le] (see, in particular, the remarks after [Le, Corollary 6.7]), part (i) of Theorem 0.4 can be strengthened to show that $R$ is an Auslander-Gorenstein, Cohen-Macaulay ring. In other words, if $M$ is a finitely generated, right $R$-module, and $N$ is a left $R$-submodule of $\operatorname{Ext}_{R}^{j}(M, R)$, for some $j$, then $\operatorname{Ext}_{R}^{i}(N, R)=0$ for all $i<j$. Moreover, if $j(M)=$ $\min \left\{j: \operatorname{Ext}_{R}^{j}(M, R) \neq 0\right\}$ is the homological grade of $M$, then $j(M)+$ $\operatorname{GKdim}(M)=\operatorname{GKdim}(R)$. By [St, Theorem 2.10], this also implies that $R$ is a maximal order in its division ring of fractions $Q(R)$; that is, if $S$ is a ring such that $R \subseteq S \subseteq Q(R)$ and $a S b \subseteq R$ for some $a, b \in R \backslash\{0\}$, then $R=S$. For the significance of these conditions the reader is referred to [Le and St].

0.6. The idea behind the proof of Theorem 0.4 is as follows. Let $T=T(V)=$ $\bigoplus T_{n}$ be the tensor algebra on $V=\sum_{0}^{3} k x_{i}$, graded in the natural way. Thus $B=T /\langle W\rangle$ and $S=T /\langle X\rangle$, where $W$ is the eight-dimensional subspace $\sum_{i} k f_{i}+k \Omega_{1}+k \Omega_{2}$ of $T_{2}=V \otimes V$ and $X$ is an appropriate six-dimensional subspace of $W$. Write $W^{\perp}$ for the orthogonal subspace to $W$ inside $V^{*} \otimes V^{*}$. Then the Koszul dual of $B$ is the algebra $B^{!}=T\left(V^{*}\right) /\left\langle W^{\perp}\right\rangle$ (just as the exterior algebra on an $n$-dimensional space $U$ is the Koszul dual of the symmetric algebra on $U)$. Given a ring $R_{Y}=T(V) /\langle Y\rangle$, for some six-dimensional subspace $Y$ of $W$, then [SS, $\S 5.6]$ lists the conditions that are required of $R_{Y}$ for it to satisfy the conclusions of Theorem 0.4 (see (1.3)). In $\S 2$, we first show that these conditions can all be rephrased in terms of properties of elements of $B$ and $B$. The most significant requirement is that $R_{Y}^{!}$should satisfy the standard properties of the exterior algebra $\Lambda\left(k^{(4)}\right)$. It is then relatively easy to find those subspaces $Y$ of $W$ for which these conditions hold. Indeed, under a reasonably mild conjecture (see (2.10)) the examples given in the theorem are the only rings $R_{Y}$ for which the theorem holds. Certainly the theorem will fail if, for example, $R_{Y}=S_{d, j}$ for the excluded values of $d$ or if $Y$ is in sufficiently general position.

In outline, the structure of the paper is as follows. In $\S 1$, we give the strategy behind the proof of Theorem 0.4 and, in particular, collect the relevant definitions and results that are needed from [SS]. $\S 2$ is devoted to the proof of Theorem 0.4 (see, in particular, (2.15)). Finally, in $\S 3$ we prove that the geometric ring $B$ is a Koszul algebra.

0.7. There is one way in which the Sklyanin algebra can be derived from its geometric ring $B$. In [OF1, 2], the authors generalize Sklyanin's construction of $S\left(\alpha_{1}, \alpha_{2}, \alpha_{3}\right)$ to provide algebras with $n \geq 3$ generators. As is the case in [Sk], these algebras are defined in terms of certain theta functions $\theta_{i}$ of order $n$. As such, an elliptic curve $E \subset \mathbb{P}^{n-1}$ and the analogue $B^{\prime}$ of the geometric ring $B$ is an integral part of their theory. Moreover, as they say in [OF2], "it is natural to ask the question how to recover a Sklyanin algebra from its characteristic variety $N \ldots$ and the bundle $\xi$ " (this data amounts to $E$ and $B$ in dimension 4). In [OF2] they do indeed show how to construct $S$ from $B$, or $X$ from $W$ in the notation of $(0.6)$, by noting that $X$ can be regarded as an appropriate space of skew-symmetric elements in $W$. As such, $X$ is not particularly canonical.

0.8. Many of the computational results of this paper were proved using Bill Schelter's Affine program and I am very grateful to him for sharing this program with me. 


\section{THE STRATEGY}

1.1. Much of the proof of Theorem 0.4 follows from the results given in [SS]. Thus, in this section we review those parts of [SS] that will be used here and, in particular, identify precisely what is required of a ring $R$ for the conclusion of Theorem 0.4 to hold. The following notation will hold throughout the paper.

Notation. The scalars $\left\{\alpha_{i}\right\}$ will always satisfy $(0.3 .1)$ and $(0.3 .3)$. Let $V$ be the four-dimensional $k$-vector space, with basis $\left\{x_{0}, x_{1}, x_{2}, x_{3}\right\}$. Write $T=T(V)=k\left\{x_{0}, x_{1}, x_{2}, x_{3}\right\}$ for the corresponding tensor algebra, with the natural grading $T=\bigoplus_{n>0} T_{n}$, where $T_{n}=V^{\otimes n}$. Let $W \subset V \otimes V$ be the space spanned by the elements $\left\{f_{1}, \ldots, f_{6}, \Omega_{1}, \Omega_{2}\right\}$, as defined by (0.3.2) and (0.4.1), and let $X=\sum_{1}^{6} f_{i} k$. Thus, in the notation of the introduction, $S=S\left(\alpha_{1}, \alpha_{2}, \alpha_{3}\right)=T(V) /\langle X\rangle$ while $B=B\left(\alpha_{1}, \alpha_{2}, \alpha_{3}\right)=$ $T(V) /\langle W\rangle$. More generally, if $Y$ is any six-dimensional subspace of $W$, set $R_{Y}=T(V) /\langle Y\rangle$. In each case of interest, the defining relations $Y$ of $R_{Y}$ will depend on $\left\{\alpha_{1}, \alpha_{2}, \alpha_{3}\right\}$, and if we need to emphasize this dependence, we will write $R_{Y}=R_{Y}\left(\alpha_{1}, \alpha_{2}, \alpha_{3}\right)$. Each of these rings has a graded structure; for example, $R_{Y}=\bigoplus_{n \geq 0}\left(R_{Y}\right)_{n}$, induced from that of $T(V)$.

1.2. The basic aim of this paper is to determine conditions on $Y$ for $R_{Y}$ to satisfy the conclusions of Theorem 0.4 . As remarked in the introduction, there is an elliptic curve $E$ and an automorphism $\tau$ of $E$ canonically associated to $S=S\left(\alpha_{1}, \alpha_{2}, \alpha_{3}\right)$ and the ring $B$ is a corresponding "twisted" homogeneous coordinate ring of $E$. These objects are, very briefly, described as follows (for further details and proofs of the statements made here, the reader is referred to [SS, §3]). Identify $S(V) \otimes S(V)=k\left[x_{01}, \ldots, x_{31}\right] \otimes k\left[x_{02}, \ldots, x_{32}\right]$ with the homogeneous coordinate ring of $\mathbb{P}^{3} \times \mathbb{P}^{3}$ in the obvious way. Similarly, we may identify $X \subset V \otimes V \subset S(V) \otimes S(V)$ and hence define $\Gamma=\Gamma\left(\alpha_{1}, \alpha_{2}, \alpha_{3}\right)$ to be the set of zeros of $X$ inside $\mathbb{P}^{3} \times \mathbb{P}^{3}$. For future reference, we note that, for any ring $R_{Y}=R_{Y}\left(\alpha_{1}, \alpha_{2}, \alpha_{3}\right)$ we may similarly define a variety $\Gamma_{Y}$ to be the zeros of $Y$ in $\mathbb{P}^{3} \times \mathbb{P}^{3}$. The variety $\Gamma$ is the union of four isolated points with $\Gamma_{1}$, where $\Gamma_{1}$ is the graph of an automorphism $\tau$ of an elliptic curve $E \subset \mathbb{P}^{3}$. Let $\mathscr{L}=i^{*} \mathscr{O}_{\mathbb{P}^{3}}(1)$ be the line bundle associated to this embedding and set $\mathscr{L}^{\tau^{n}}=\left(\tau^{n}\right)^{*} \mathscr{L}$, the inverse image of $\mathscr{L}$ along $\tau^{n}: E \rightarrow E$. Write $\mathscr{L}_{n}=\mathscr{L} \otimes \cdots \otimes \mathscr{L}^{\tau^{n-1}}$, where $\mathscr{L}_{0}=\mathscr{O}_{E}$. Then $B=\bigoplus B_{n}$, where $B_{0}=k$ and $B_{n}=H^{0}\left(E, \mathscr{L}_{n}\right)$. The multiplication map $B_{n} \times B_{m} \rightarrow B_{n+m}$ is defined by taking global sections of the natural isomorphism $\mathscr{L}_{n} \otimes \mathscr{L}_{m}^{\tau^{n}} \rightarrow \mathscr{L}_{n+m}$. The advantage of this construction is that standard algebreogeometric results may be used to prove strong results about the structure of $B$. For example, [SS, Theorems 3.4 and 5.5] show that $B$ is a Noetherian domain, $\operatorname{dim}_{k}\left(B_{n}\right)=4 n$, for all $n \geq 1$ and the $\operatorname{socle}, \operatorname{Soc}(B / g B)$ is zero for all $g \in B$. Moreover, $B$ is indeed a factor ring of $S$. Once again, these objects depend upon the $\alpha_{j}$ and when we need to emphasize this dependence we will write, for example, $E=E\left(\alpha_{1}, \alpha_{2}, \alpha_{3}\right)$ or $\Gamma_{Y}=\Gamma_{Y}\left(\alpha_{1}, \alpha_{2}, \alpha_{3}\right)$, etc.

In order to use this information to understand $S$ and $R_{Y}$ we need the concept of a Koszul complex. Let $Z \subseteq V \otimes V$ and $A=A_{Z}=T(V) /\langle Z\rangle$ be the corresponding quadratic algebra. Let $\left\{\xi_{0}, \xi_{1}, \xi_{2}, \xi_{3}\right\}$ be the basis of $V^{*}$ dual to the basis $\left\{x_{i}\right\}$ of $V$ and write $Z^{\perp}$ for the orthogonal to $Z$ in $V^{*} \otimes V^{*}$. Define $A^{!}=T\left(V^{*}\right) /\left\langle W^{\perp}\right\rangle$, which again inherits a natural graded 
structure, $A^{!}=\bigoplus_{n \geq 0} A_{n}^{!}$, from $T\left(V^{*}\right)$. Associated to $A$ and $A^{!}$are two Koszul complexes. Set $e=\sum_{j=0}^{3} x_{j} \otimes \xi_{j} \in A_{1} \otimes A_{1}^{!}$and note that $e^{2}=0$ [Ma, Lemma 9.1]. Define $K^{\bullet}=\bigoplus_{n \geq 0} K^{n}$, where $K^{n}=A \otimes A_{n}^{!}$, and let $d^{*}: K^{n} \rightarrow K^{n+1}$ denote left multiplication by $e$. Since $e^{2}=0,\left(K^{\bullet}, d^{*}\right)$ is a complex of free right $A$-modules, called a Koszul complex. The second complex of interest is the dual of $\left(K^{\bullet}, d^{*}\right)$, namely the Koszul complex of free left $A$ modules

$$
\left(K_{\bullet}, d\right)=\left(K_{\bullet}(A), d\right)=\operatorname{Hom}_{A}\left(\left(K^{\bullet}, d^{*}\right), A\right) .
$$

More explicitly, the left regular representation of $A^{!}$on itself induces a right $A^{!}$-module structure on $\left(A^{!}\right)^{*}=\bigoplus_{n \geq 0}\left(A_{n}^{!}\right)^{*}$. Thus, if $K_{n}=A \otimes\left(A_{n}^{!}\right)^{*}$ and $K_{\bullet}=\bigoplus_{n \geq 0} K_{n}$, then right multiplication by $e$ induces a map $d: K_{n} \rightarrow K_{n-1}$ and hence a differential $d: K_{\bullet} \rightarrow K_{\bullet}$.

For any quadratic algebra $A$, the Koszul dual $A^{!}$is isomorphic to the subalgebra of the Yoneda Ext algebra $\operatorname{Ext}_{A}^{*}(k, k)$ generated by $\operatorname{Ext}_{A}^{1}(k, k)$. Moreover, $A$ is Koszul if and only if the augmented complex $\left(K_{\bullet}(A), d\right) \rightarrow k \rightarrow 0$ is exact (see, for example, [Lo, Theorem 1.2]).

1.3. Now suppose that $Y$ is a six-dimensional subspace of $W$. Then the identification $\left(R_{Y}\right)_{1}=V=B_{1}$ induces a surjection $\phi: R_{Y} \rightarrow B$. Dually, the identification $\left(R_{Y}^{!}\right)_{1}=B_{1}^{!}$induces a surjection $\phi^{!}: B^{!} \rightarrow R_{Y}^{!}$. The proof of Theorem 0.4 in [SS] depends upon an examination of the homology groups of $\left(K_{\bullet}(S), d\right)$ and certain other complexes. However, this proof does not require the explicit description of $S$. Rather, it requires that $R=S$ satisfies the following four properties:

(1.3.1) $R^{!}$has the same Hilbert series as the exterior algebra $\Lambda\left(k^{(4)}\right)$; that is, $\operatorname{dim}\left(R^{!}\right)_{n}=1,4,6,4,1,0,0, \ldots$ for $n=0,1,2, \ldots$

(1.3.2) $B=R / \Psi_{1} R+\Psi_{2} R$, where $\left\{\Psi_{1}, \Psi_{2}\right\}$ is a normalizing sequence of elements of $R$; that is, $\Psi_{1} R=R \Psi_{1}$ and $\Psi_{2} \bar{R}=\bar{R} \Psi_{2}$, where $\bar{R}=$ $R / \Psi_{1} R$.

(1.3.3) The left-hand end

$$
0 \longrightarrow B \otimes\left(R^{!}\right)_{4}^{*} \stackrel{d}{\longrightarrow} B \otimes\left(R^{!}\right)_{3}^{*} \stackrel{d}{\longrightarrow} B \otimes\left(R^{!}\right)_{2}^{*}
$$

of the complex $B \otimes\left(K_{\bullet}(R), d\right)$ is exact.

(1.3.4) $R^{!}$is Frobenius; that is $\left(R^{!} R^{!}\right)^{*} \cong R_{R^{!}}^{!}$, as right $R^{!}$-modules.

(1.3.5) There exists an antiautomorphism $\sigma$ of $R$ such that, if the induced antiautomorphisms of $R^{!}$and $R \otimes R^{!}$are still denoted by $\sigma$, then $\sigma$ fixes the elements $x_{j} \otimes \xi_{j} \in R \otimes R^{!}$.

It follows that the proof of [SS, Theorem 5.4 and 5.5] can also be used to prove the following result (see [SS, §5.6]):

Theorem. Suppose $\left\{\alpha_{i}\right\}$ satisfy (0.3.1) and (0.3.3) and that $R_{Y}=T(V) /\langle Y\rangle$, for some six-dimensional subspace $Y$ of $W$. Assume that $R_{Y}$ satisfies (1.3.1)(1.3.5). Then:

(i) $H_{R_{Y}}(t)=1 /(1-t)^{4}$.

(ii) $R_{Y}$ is a Noetherian domain.

(iii) $R_{Y}$ is a regular graded ring of dimension 4 .

(iv) $R_{Y}$ is Koszul. 
(v) $\left\{\Psi_{1}, \Psi_{2}\right\}$ is a regular sequence in the sense that $\Psi_{1}$ is a non-zero-divisor in $R_{Y}$ while $\Psi_{2}$ is a non-zero-divisor in $R_{Y} / \Psi_{1} R_{Y}$.

Proof. To be more precise, the proof of [SS, Theorems 5.4 and 5.5] show that (1.3.1), (1.3.2), and (1.3.3) are sufficient to imply parts (i), (iv), and (v) of the theorem, and to prove that $R_{Y}$ is a Noetherian ring of finite global dimension. The conditions (1.3.4) and (1.3.5) imply that the two complexes $\left(K^{\bullet}\left(R_{Y}\right), d^{*}\right)$ and $\left(K_{\bullet}\left(R_{Y}\right), d\right)$ are isomorphic as complexes of vector spaces. It follows that, since $R_{Y}$ is Koszul, it is Gorenstein (see [SS, Proposition 4.10]). Finally, [ATV2, Theorem 3.9] can then be used to prove that $R_{Y}$ is a domain.

\section{THE MAIN RESULT}

2.1. In this section we find subspaces $Y \subset W$ such that the conditions (1.3.1)(1.3.5) hold for the ring $R_{Y}$ and hence such that Theorem 0.4 applies to $R_{Y}$. We will first show that condition (1.3.2) can also be phrased in terms of $B^{!}$. Indeed, in the presence of condition (1.3.1), it is equivalent to the statement that $R_{Y}^{!}=B^{!} / a B^{!}+b B^{!}$for a 1-regular, normalizing sequence $\{a, b\} \in B_{2}^{!}$ (see 2.6 and 2.8 for the precise statement). Moreover, (1.3.1) and (1.3.2) are in some sense the key requirements since the other three conditions will hold in each case when (1.3.1) and (1.3.2) are true. Thus, most of this section will be concerned with the study of $B^{!}$and we begin with two elementary results to give the reader a feel for the structure of this ring. The first one is routine.

2.2. Given elements $a, b$ in a ring $R$, write $[a, b]=a b-b a$ and $[a, b]_{+}=$ $a b+b a$.

Lemma. The vector space $W^{\perp}$ has basis

$$
\begin{aligned}
w_{i} & =\left[\xi_{0}, \xi_{i}\right]+\frac{1}{\alpha_{i}}\left[\xi_{i+1}, \xi_{i+2}\right]_{+} \text {for } 1 \leq i \leq 3, \\
w_{i+3} & =\left[\xi_{0}, \xi_{i}\right]_{+}+\left[\xi_{i+1}, \xi_{i+2}\right] \text { for } 1 \leq i \leq 3, \\
w_{7} & =\left(\frac{1-\alpha_{1}}{1+\alpha_{3}}-1\right) \xi_{0}^{2}+\frac{1-\alpha_{1}}{1+\alpha_{3}} \xi_{1}^{2}-\xi_{3}^{2}, \\
w_{8} & =\left(\frac{1+\alpha_{1}}{1-\alpha_{2}}-1\right) \xi_{0}^{2}+\frac{1+\alpha_{1}}{1-\alpha_{2}} \xi_{1}^{2}-\xi_{2}^{2} .
\end{aligned}
$$

Here, $\xi_{3+j}=\xi_{j}$ for $j>0$.

2.3. Clearly, an element $y \in B_{n}^{!}$is normal if and only if there exists a basis $\left\{r_{0}, r_{1}, r_{2}, r_{3}\right\}$ of $B_{1}^{\prime}$ such that $\xi_{i} y=y r_{i}$ for each $i$. This will be written $\left(\xi_{0}, \ldots, \xi_{3}\right)^{y}=\left(r_{0}, \ldots, r_{3}\right)$.

Proposition. Set

$$
\begin{array}{lll}
t_{1}=\xi_{0}^{2}, & t_{2}=\xi_{1}^{2}, & t_{3}=\xi_{0} \xi_{1}-\xi_{1} \xi_{0}, \\
t_{4}=\xi_{0} \xi_{1}+\xi_{1} \xi_{0}, & t_{5}=\xi_{0} \xi_{2}-\xi_{2} \xi_{0}, & t_{6}=\xi_{0} \xi_{2}+\xi_{2} \xi_{0}, \\
t_{7}=\xi_{0} \xi_{3}-\xi_{3} \xi_{0}, & t_{8}=\xi_{0} \xi_{3}+\xi_{3} \xi_{0} . &
\end{array}
$$

Then:

(i) $\left\{t_{1}, \ldots, t_{8}\right\}$ form a basis of $B !$. 
(ii) Moreover, $t_{1}$ and $t_{2}$ are central, while $t_{3}, \ldots, t_{8}$ are normal. If $3 \leq$ $j \leq 8$, then $\left(\xi_{0}, \ldots, \xi_{3}\right)^{t_{j}}$ is the $(j-2)$ th row of the matrix

$$
\left(\begin{array}{cccc}
- & - & + & + \\
+ & + & - & - \\
- & + & - & + \\
+ & - & + & - \\
- & + & + & - \\
+ & - & - & +
\end{array}\right)\left(\begin{array}{cccc}
\xi_{0} & 0 & 0 & 0 \\
0 & \xi_{1} & 0 & 0 \\
0 & 0 & \xi_{2} & 0 \\
0 & 0 & 0 & \xi_{3}
\end{array}\right) .
$$

(iii) Finally, $\operatorname{dim}_{k} B_{n}^{!}=4 n$ for $1 \leq n$.

Proof. This was proved using Schelter's Affine program. However, parts (i) and (ii) of the proposition are easy enough to prove directly while an alternative, more theoretical proof of part (iii) is given by Theorem 3.9.

2.4. The defining relations $W^{\perp}$ of $B^{!}$are, at first glance, very similar to those of $B$, since they amount to no more than a slight change of the scalars in those relations. Yet the structures of $B$ and $B^{!}$are very different. For example, at least for sufficiently general values of the $\left\{\alpha_{i}\right\}$, there are no central elements in $B_{2}$. We know of no good theoretical reasons why this should be true (but see 2.7). Possibly a more intuitive proof of part (ii) of the proposition can be obtained by regarding $B^{!}$as $\operatorname{Ext}_{B}^{*}(k, k)$ and using the geometric techniques of, for example, [SS, §3]. It would also be more satisfactory if similarly less computational proofs could be found for some of the other results of this section, notably 2.12 and 2.14 .

Finally, we conjecture that the only normalizing sequences $\{a, b\}$ of elements of $B^{!}$are those for which $a, b \in\left\{d_{1} t_{1}+d_{2} t_{2}, t_{3}, \ldots, t_{8}: d=\left(d_{1}, d_{2}\right) \in\right.$ $\left.\mathbb{P}_{k}^{1}\right\}$.

2.5. We next show how to express condition (1.3.2) in terms of $B^{!}$, by relating properties of the kernels of the two surjections $R_{Y} \rightarrow B$ and $B^{!} \rightarrow R_{Y}^{!}$. We begin with an abstract result.

Lemma. Let $R=\bigoplus R_{i}$ be a quadratic algebra, say $R=T(F) /\langle G\rangle$, where $F=R_{1}$ is an n-dimensional $k$-vector space and $G \subset F \otimes F$. Let $y \in F \otimes F \backslash G$ and set $H=G+k y$. Write $P=R / R y R=T(F) /\langle H\rangle$ and $G^{\perp} / H^{\perp}=k \bar{\eta}$, where $\bar{\eta}=\left[\eta+H^{\perp}\right]$, for some $\eta \in G^{\perp}$. Then the following are equivalent:

(i) The element $y$ is normal in $R$.

(ii) If $P^{!}=T\left(F^{*}\right) /\left\langle H^{\perp}\right\rangle$, then $\operatorname{dim}_{k}\left(P_{1}^{!} \bar{\eta}\right)=n=\operatorname{dim}_{k}\left(\bar{\eta} P_{1}^{!}\right)$.

Proof. All computations will take place inside (factors of) $T(F)$ and $T\left(F^{*}\right)$. Observe that, since $\eta \notin H^{\perp}$, one has

$$
n=\operatorname{dim}_{k}\left(F^{*} \otimes G^{\perp} / F^{*} \otimes H^{\perp}\right)=\operatorname{dim}_{k}\left(G^{\perp} \otimes F^{*} / H^{\perp} \otimes F^{*}\right) .
$$

Moreover,

$$
\begin{aligned}
P_{i}^{!} \bar{\eta} & \cong\left(F^{*} \otimes \eta+F^{*} \otimes H^{\perp}+H^{\perp} \otimes F^{*}\right) /\left(F^{*} \otimes H^{\perp}+H^{\perp} \otimes F^{*}\right) \\
& =\left(F^{*} \otimes G^{\perp}+H^{\perp} \otimes F^{*}\right) /\left(F^{*} \otimes H^{\perp}+H^{\perp} \otimes F^{*}\right),
\end{aligned}
$$

and, similarly, $\bar{\eta} P_{1}^{!} \cong\left(F^{*} \otimes H^{\perp}+G^{\perp} \otimes F^{*}\right) /\left(F^{*} \otimes H^{\perp}+H^{\perp} \otimes F^{*}\right)$. Bearing (2.5.1) in mind, this means that we have the following chain of equivalent 
conditions:

condition (ii) holds

$\Leftrightarrow$ the natural surjections $\theta_{1}: F^{*} \otimes G^{\perp} / F^{*} \otimes H^{\perp} \rightarrow P_{1}^{!} \eta$ and

$\theta_{2}: G^{\perp} \otimes F^{*} / H^{\perp} \otimes F^{*} \rightarrow \eta P_{1}^{!}$are isomorphisms

$\Leftrightarrow \frac{F^{*} \otimes G^{\perp} \cap H^{\perp} \otimes F^{*}}{F^{*} \otimes H^{\perp} \cap H^{\perp} \otimes F^{*}} \cong \operatorname{Ker} \theta_{1}=0=\operatorname{Ker} \theta_{2} \cong \frac{G^{\perp} \otimes F^{*} \cap F^{*} \otimes H^{\perp}}{H^{\perp} \otimes F^{*} \cap F^{*} \otimes H^{\perp}}$

$\Leftrightarrow F^{*} \otimes G^{\perp} \cap H^{\perp} \otimes F^{*}=G^{\perp} \otimes F^{*} \cap F^{*} \otimes H^{\perp}$

$\Leftrightarrow(F \otimes G+H \otimes F)^{\perp}=(G \otimes F+F \otimes H)^{\perp}$

$\Leftrightarrow F \otimes G+H \otimes F=G \otimes F+F \otimes H$

$\Leftrightarrow \frac{F \otimes G+H \otimes F}{F \otimes H+H \otimes F}=\frac{G \otimes F+F \otimes H}{F \otimes H+H \otimes F}$

$\Leftrightarrow R_{1} y=y R_{1}$

$\Leftrightarrow y$ is normal in $R$.

2.6. Motivated by Lemma 2.5, we make the following definition. Let $R=$ $\bigoplus R_{n}$ be a graded ring and let $y \in R_{r}$, for some $r$. Then $y$ is called $n$-regular if $t=0$ is the only element $t \in \bigoplus_{m \leq n} R_{m}$ such that $t y=0$ or $y t=0$. Of course, $y$ is 1-regular if and only if $\operatorname{dim}_{k}\left(R_{1} y\right)=\operatorname{dim}_{k}\left(R_{1}\right)=\operatorname{dim}_{k}\left(y R_{1}\right)$. Similarly, a pair $\{a, b\} \in R_{r}$ is called an n-regular (normalizing) sequence if $a$ is $n$-regular (and normalizing) in $R$ while $b$ is $n$-regular (and normalizing) in $R / R a R$.

Corollary. Let $Y$ be a six-dimensional subspace of $W$. Then $B \cong R_{Y} / \Psi_{1} R_{Y}+$ $\Psi_{2} R_{Y}$, for some normalizing sequence $\left\{\Psi_{i}\right\}$ if and only if

$$
R_{Y}^{!} \cong B^{!} /\left(B^{!} a B^{!}+B^{!} b B^{!}\right)
$$

for some 1-regular sequence $\{a, b\} \in B_{2}^{!}$.

2.7 Remark. Lemma 2.5 actually forces $B^{!}$to contain normal elements. For, [SS, Theorem 5.4(iii)] implies that $B \cong S / \Omega_{1} S+\Omega_{2} S$, where $\Omega_{1}$ is 1-regular in $S$ and $\Omega_{2}$ is 1-regular in $S / \Omega_{1} S$. However, we feel that this is not a satisfactory answer to the question raised in 2.4 .

2.8. When combined with condition (1.3.1), Corollary 2.6 places considerable restrictions on the possible choices of $Y$. For, suppose that a six-dimensional subspace $Y$ of $W$ is chosen such that (1.3.1) and (1.3.2) both hold, and keep the notation of Corollary 2.6. Write $\bar{B} !=B^{!} / B^{!} a B^{!}$. Then

$$
\begin{aligned}
4 & =\operatorname{dim}_{k}\left(R_{Y}^{!}\right)_{3}=\operatorname{dim} \bar{B}_{3}^{!}-\operatorname{dim}\left(\bar{B}_{1}^{!} b+b \bar{B}_{1}^{!}\right) \\
& \leq \operatorname{dim} \bar{B}_{3}^{!}-\operatorname{dim} \bar{B}_{1} b=\operatorname{dim} \bar{B}_{3}-4
\end{aligned}
$$

and

$$
\begin{aligned}
\operatorname{dim} \bar{B}_{3}^{!} & =\operatorname{dim} B_{3}^{!}-\operatorname{dim}\left(B_{1}^{!} a+a B_{1}^{!}\right) \\
& \leq \operatorname{dim} B_{3}^{!}-\operatorname{dim} B_{1}^{!} a=12-4=8 .
\end{aligned}
$$


By comparing these two equations, one sees that both inequalities are actually equalities. This in turn implies that $B_{1}^{!} a=B_{1}^{!} a+a B_{1}^{!}=a B_{1}^{!}$and $\bar{B} ! b=b \bar{B}_{1}^{!}$. In other words, $\{a, b\}$ is a normal sequence. This proves one half of the next result, the other half being proved by reversing the argument given above.

Lemma. Let $Y$ be a six-dimensional subspace of $W$. Then $R_{Y}$ satisfies $\operatorname{dim}_{k}\left(R_{Y}^{!}\right)_{3}=4$ and (1.3.2) if and only if $R_{Y}^{!}=B^{!} / a B^{!}+b B^{!}$for some 1regular normalizing sequence of elements $\{a, b\} \subset B_{2}^{!}$.

2.9 Corollary. Suppose that $Y$ is a six-dimensional subspace of $W$. Assume that, if $R_{Y}^{!}=B^{!} / B^{!} a B^{!}+B^{!} b B^{!}$, for $a, b \in B_{2}^{!}$, then $a$ and $b$ are 3-regular. Then the following are equivalent:

(i) $R_{Y}$ satisfies (1.3.1) and (1.3.2).

(ii) $\{a, b\}$ is a normalizing sequence and $a B_{r}^{!} \cap b B_{r}^{!}=b a B_{r-2}^{!}$for $r \leq 3$ (where $B_{j}^{!}=0$ if $j<0$ ).

Proof. This exercise in dimension counting is left to the reader.

2.10. It is not clear whether the hypothesis of Corollary 2.9 is ever satisfied, but that is not the point of the result. Rather, it has been included in order to explain why the next lemma should be expected.

Thus, we wish to find $a, b \in B_{2}^{!}$such that the six-dimensional subspace $Y \subset W$, defined by $R_{Y}^{!}=B^{!} / B^{!} a B^{!}+B^{!} b B^{!}$, will satisfy (1.3.1) and (1.3.2). By Lemma 2.8, we need to assume that $\{a, b\}$ is a normalizing sequence. To the best of our knowledge this forces $\{a, b\} \in\left\{d_{1} t_{1}+d_{2} t_{2}, t_{3}, \ldots, t_{8}\right.$ : $\left.d=\left(d_{1}, d_{2}\right) \in \mathbb{P}_{k}^{1}\right\}$. Moreover, each of these elements can be shown to be 3-regular. Of course, if one chooses two normal elements $a, b \in B_{2}^{!}$that are in "sufficiently general position" then one would expect $a B^{!} \cap b B^{!}=a b B^{!}$to hold and so Corollary 2.9 would imply that the corresponding ring $R_{Y}$ would indeed satisfy (1.3.1) and (1.3.2). Given the nature of the normal elements in $B_{2}^{!}$, the nearest approximation to general position that we have is for $a=t_{j}$, for some $j \geq 3$ and $b=t_{1}+d_{2} t_{2}$, for $d_{2}$ in some Zariski open subset of $k$. While this does not provide a proof, it does at least indicate that some version of the next result is to be expected.

Proposition. The ring $R_{Y}^{!}=B^{!} / a B^{!}+b B^{!}$satisfies (1.3.1) and (1.3.2) in the following cases:

(i) $a=t_{3}$ and $b=d_{1} t_{1}+d_{2} t_{2}$ for $d=\left(d_{1}, d_{2}\right) \in \mathbb{P}_{k}^{1}$ provided that $d \neq$ $(1,1),\left(1,-1 / \alpha_{2} \alpha_{3}\right)$;

(ii) $a=t_{4}$ and $b=d_{1} t_{1}+d_{2} t_{2}$ for $d=\left(d_{1}, d_{2}\right) \in \mathbb{P}_{k}^{1}$ provided that $d \neq$ $\left(1,1 / \alpha_{2}\right),\left(1,-1 / \alpha_{3}\right)$

(iii) $a=t_{1}$ and $b=t_{2}$; or

(iv) $a=t_{3}$ and $b=t_{4}$.

Up to isomorphisms of the $R_{Y}^{!}$given by permutation of the variables $\left\{x_{1}, x_{2}\right.$, $\left.x_{3}\right\}$, these are the only choices of $a, b \in\left\{d_{1} t_{1}+d_{2} t_{2}, t_{3}, \ldots, d_{8}: d=\left(d_{1}, d_{2}\right) \in\right.$ $\left.\mathbb{P}_{k}^{1}\right\}$ for which the corresponding ring $R_{Y}^{!}=B^{!} / a B^{!}+b B^{!}$satisfies (1.3.1) and (1.3.2).

Proof. Suppose that $a, b \in B_{2}^{!}$are defined by (i)-(iv) of the lemma. Then, by Corollary 2.6 , we need to prove that $\{a, b\}$ is a 1 -regular sequence and 
that the corresponding ring $R_{Y}^{!}$satisfies (1.3.1). This has been proved using Schelter's Affine program.

To prove the result stated in the second paragraph of the proposition, we should first be precise about the isomorphisms involved. The basic observation is that cyclically permuting the variables $\left\{x_{1}, x_{2}, x_{3}\right\}$ produces isomorphisms $B^{!}\left(\alpha_{1}, \alpha_{2}, \alpha_{3}\right) \cong B^{!}\left(\alpha_{2}, \alpha_{3}, \alpha_{1}\right) \cong B^{!}\left(\alpha_{3}, \alpha_{1}, \alpha_{2}\right)$ and this induces isomorphisms between various of the $R_{Y}^{!}$(and hence between various of the $R_{Y}$ ). In particular, if $n=2$ or 3 then

$$
\begin{aligned}
B^{!}\left(\alpha_{1}, \alpha_{2}, \alpha_{3}\right) /\left(t_{n}, t_{1}+d t_{2}\right) & \cong B^{!}\left(\alpha_{2}, \alpha_{3}, \alpha_{1}\right) /\left(t_{n+2}, t_{1}+d^{\prime} t_{2}\right) \\
& \cong B^{!}\left(\alpha_{3}, \alpha_{1}, \alpha_{2}\right) /\left(t_{n+4}, t_{1}+d^{\prime \prime} t_{2}\right),
\end{aligned}
$$

where $d^{\prime}$ and $d^{\prime \prime}$ can be computed from Lemma 2.2; for example,

$$
d^{\prime}=\left(d \frac{1-\alpha_{1}}{1+\alpha_{3}}\right) /\left(1-d+d \frac{1-\alpha_{1}}{1+\alpha_{3}}\right) \text {. }
$$

Similarly,

$$
\begin{aligned}
B^{!}\left(\alpha_{1}, \alpha_{2}, \alpha_{3}\right) /\left(t_{3}, t_{4}\right) & \cong B^{!}\left(\alpha_{2}, \alpha_{3}, \alpha_{1}\right) /\left(t_{5}, t_{6}\right) \\
& \cong B^{!}\left(\alpha_{3}, \alpha_{1}, \alpha_{2}\right) /\left(t_{7}, t_{8}\right) .
\end{aligned}
$$

Given any choice of $\{a, b\} \in\left\{d_{1} t_{1}+d t_{2}, t_{3}, \ldots, t_{8}: d=\left(d_{1}, d_{2}\right) \in \mathbb{P}_{k}^{1}\right\}$, other than those covered by parts (i)-(iv) of the proposition and their images under the isomorphisms given above, then the same $A$ ffine program can be used to prove that $\operatorname{dim}\left(R_{Y}^{!}\right)_{n}>0$ for $n \geq 5$.

2.11 Remarks. We will let $\mathscr{Y}$ denote the class of rings defined by parts (i)-(iv) of the proposition .

Since $\left(R_{Y}^{!}\right)^{!}=R_{Y}$, it is easy to check that these rings are precisely the rings defined in the first two paragraphs of Theorem 0.4. Specifically:

(i) If $a=t_{j}$ for $j \geq 3$ and $b=d_{1} t_{1}+d_{2} t_{2}$, for $d=\left(d_{1}, d_{2}\right) \in \mathbb{P}_{k}^{1}$, then $R_{Y}=S_{e, j-2}$ for $e=\left(d_{2}, d_{1}-d_{2}\right) \in \mathbb{P}_{k}^{1}$.

(ii) If $a=t_{1}$ and $b=t_{2}$, then $R_{Y}=S$, the Sklyanin algebra.

(iii) If $a=t_{3}$ and $b=t_{4}$, then $R_{Y}=S_{\infty}$.

It is readily checked that the excluded cases of parts (i) and (ii) of the proposition are in agreement with the excluded cases among the $S_{e, j}$ of Theorem 0.4 .

2.12 Lemma. For each ring $R_{Y} \in \mathscr{Y}$, the Koszul dual $R_{Y}^{!}$is Frobenius.

Proof. Fix $\eta \in\left(R_{Y}^{!}\right)_{4} \backslash\{0\}$. Given $u, v \in R_{Y}^{!}$, write $u v=\lambda \eta+h$, for some $h \in \bigoplus_{m \leq 3}\left(R_{Y}^{!}\right)_{m}$ and set $\Phi(u, v)=\lambda$. Then $R_{Y}^{!}$is Frobenius provided that $\Phi$ is nondegenerate. Once again, explicit computations show this to be the case.

\subsection{Lemma. Let $R_{Y} \in \mathscr{Y}$. Then $R_{Y}$ satisfies condition (1.3.5).}

Proof. The antiautomorphism is the same one that was used in [SS]; viz, the map defined by $\sigma\left(x_{0}\right)=-x_{0}$ and $\sigma\left(x_{j}\right)=x_{j}$ for $j \neq 0$. By duality, $\sigma$ induces an involutive antiautomorphism of $R_{y}^{!}$, which is also denoted by $\sigma$; that is $\sigma\left(\xi_{0}\right)=-\xi_{0}$, and $\sigma\left(\xi_{i}\right)=\xi_{i}$ for $i \neq 0$. This induces an anti-automorphism, still denoted by $\sigma$, of $R_{y} \otimes R_{y}^{!}$. Clearly, $\sigma(e)=e$, where $e=\sum x_{j} \otimes \xi_{j} \in$ $R_{Y} \otimes R_{Y}^{!}$. 
2.14. Let $R_{Y} \in \mathscr{Y}$. We wish to show that condition (1.3.3) holds for $R_{Y}$ and we will prove this by reducing to a case that is essentially covered by [SS, Proposition 5.3]. Now $R_{Y}^{!}$is Frobenius, by Lemma 2.12. Thus the complex $\left(K_{\bullet}\left(R_{Y}\right), d\right)$ is isomorphic to the complex of left $R_{Y}$-modules:

$$
0 \longrightarrow R_{Y} \otimes\left(R_{Y}^{!}\right)_{0} \stackrel{d_{1}}{\longrightarrow} R_{Y} \otimes\left(R_{Y}^{!}\right)_{1} \stackrel{d_{2}}{\longrightarrow} R_{Y} \otimes\left(R_{Y}^{!}\right)_{2} \stackrel{d_{3}}{\longrightarrow} \cdots
$$

where, again, each map $d_{i}$ is given by right multiplication by $e=\sum x_{j} \otimes \xi_{j}$ (see the proof of [SS, Proposition 4.10]).

Write $L=B_{2}^{!} /\left(t_{1} k+t_{2} k+t_{3} k+t_{4} k\right)=B_{2}^{!} /\left(\xi_{0}^{2} k+\xi_{1}^{2} k+\xi_{0} \xi_{1} k+\xi_{1} \xi_{0} k\right)$. Since $R_{Y} \in \mathscr{Y}$, the natural surjection $\pi: B_{2}^{!} \rightarrow L$ factors; $B_{2}^{!} \stackrel{\pi_{Y}}{\longrightarrow}\left(R_{Y}^{!}\right)_{2} \stackrel{\rho_{Y}}{\longrightarrow} L$. When $R_{Y}=S$, we will write $\rho_{S}$ for $\rho_{Y}$. Thus, in order to prove (1.3.3), it certainly suffices to show that the sequence

$$
0 \longrightarrow B \otimes\left(R_{Y}^{!}\right)_{0} \stackrel{d_{1}}{\longrightarrow} B \otimes\left(R_{Y}^{!}\right)_{1} \stackrel{\rho_{Y} d_{2}}{\longrightarrow} B \otimes L
$$

is exact. But this sequence is independent of $Y$. For, under the natural identifications $\left(R_{Y}^{!}\right)_{0}=B_{0}^{!}$and $\left(R_{Y}^{!}\right)_{1}=B_{1}^{!}, d_{1}$ is unchanged while $\rho_{Y} d_{2}$ is identified with the map $\pi d_{2}: B \otimes B_{1}^{!} \rightarrow B \otimes B_{2}^{!} \rightarrow B \otimes L$. Thus, it suffices to prove that the sequence

$$
0 \longrightarrow B \otimes S_{0}^{!} \stackrel{d_{1}}{\longrightarrow} B \otimes S_{1}^{!} \stackrel{\rho_{S} d_{2}}{\longrightarrow} B \otimes L
$$

is exact.

This is, essentially, what is proved in [SS, Proposition 5.3] in proving that the sequence $0 \longrightarrow B \otimes S_{0}^{!} \stackrel{d_{1}}{\longrightarrow} B \otimes S_{1}^{!} \stackrel{d_{2}}{\longrightarrow} B \otimes S_{2}^{!}$is exact. In the proof of that result, one first constructs a matrix $N$ such that $d_{2}$ is given by right multiplication by $N$, corresponding to the ordered bases $\left\{\xi_{0}, \xi_{1}, \xi_{2}, \xi_{3}\right\}$ of $S_{1}^{!}$and $\left\{\xi_{0} \xi_{1}, \xi_{0} \xi_{2}, \xi_{0} \xi_{3}, \xi_{1} \xi_{0}, \xi_{2} \xi_{0}, \xi_{3} \xi_{0}\right\}$ of $S_{2}^{!}$(see [SS, Lemma 4.11]). But the proof of [SS, Proposition 5.3] only used properties of the second, fifth, and sixth columns of $N$. Moreover, the choice of $L$ ensures that the matrix corresponding to the map $\rho_{S} d_{2}$ is the $4 \times 4$ matrix obtained by deleting columns 1 and 4 from $N$. Therefore, the proof given in [SS] can also be used to prove that $(2.14 .1)$ is exact. This proves:

Lemma. Let $R_{Y} \in \mathscr{Y}$. Then (1.3.3) holds for $R_{Y}$; that is, the left-hand end

$$
0 \longrightarrow B \otimes\left(R_{Y}^{!}\right)_{4}^{*} \stackrel{d}{\longrightarrow} B \otimes\left(R_{Y}^{!}\right)_{3}^{*} \stackrel{d}{\longrightarrow} B \otimes\left(R_{Y}^{!}\right)_{2}^{*}
$$

of the complex $B \otimes\left(K_{\bullet}\left(R_{Y}\right), d\right)$ is exact.

2.15. By combining (2.10)-(2.14) with Theorem 1.3 , we have proved parts (i)-(iv) of Theorem 0.4. The rest of that theorem follows from the next result.

Lemma. Let $\left\{\alpha_{i}\right\},\left\{\beta_{i}\right\}$, and $\left\{\gamma_{i}\right\}$ satisfy (0.3.1) and (0.3.3). Then there exists no isomorphism between the three rings $S\left(\alpha_{1}, \alpha_{2}, \alpha_{3}\right), S_{d, i}\left(\beta_{1}, \beta_{2}, \beta_{3}\right)$, and $S_{\infty}\left(\gamma_{1}, \gamma_{2}, \gamma_{3}\right)$.

Remark. For sufficiently general $\alpha_{i}, \beta_{j}$ and $\gamma_{k}$, an alternative proof may be obtained from the comments given below in (2.20).

Proof. (i) Suppose that there exists an isomorphism $\psi: S_{d, i} \rightarrow S$ (or $\psi: S_{\infty} \rightarrow$ $S)$ and write $\psi\left(x_{j}\right)=s_{j}=\mu_{j}+r_{j}+h_{j}$, for $\mu_{j} \in S_{0}, r_{j} \in S_{1}$ and $h_{j} \in \bigoplus_{m \geq 2} S_{m}$. As the $s_{j}$ generate $S$, certainly the $r_{j}$ are linearly independent. Now, the $s_{j}$ 
satisfy (at least) four of the relations $f_{i}$ defined in (0.3.2). Write out these equations and look at their components in $S_{0}$ and $S_{1}$. Then there is ample information to prove that $\mu_{j}=0$ for each $j$. Next, the $s_{j}$ satisfy $\sum_{0}^{3} \lambda_{j} s_{j}^{2}=0$ for some $\lambda_{j} \in k$ not all of which are zero. By looking at the component of this equation in $S_{2}$ one finds that $\sum_{0}^{3} \lambda_{j} r_{j}^{2}=0$. The proof will be completed by showing that this leads to a contradiction.

Write $r_{i}=\sum_{j} a_{i j} x_{j}$ for some $a_{i j} \in k$ and let $A=\left(a_{i j}\right)$ be the corresponding matrix. Write $\Lambda$ for the diagonal matrix $\operatorname{diag}\left\{\lambda_{0}, \lambda_{1}, \lambda_{2}, \lambda_{3}\right\}$, let $\mathbf{x}$ denote the column vector $\left(x_{0}, x_{1}, x_{2}, x_{3}\right)^{T}$, and set $D=A^{T} \Lambda A=\left(d_{i j}\right)$. Then, the equation $\sum_{0}^{3} \lambda_{j} r_{j}^{2}=0$ implies that $\sum \lambda_{i} a_{i j} a_{i k} x_{j} x_{k}=0$. Equivalently,

$$
0=\sum_{i, j} d_{i j} x_{i} x_{j}=\sum_{k} d_{k k} x_{k}^{2}+\sum_{k<l} d_{k l}\left(x_{k} x_{l}+x_{l} x_{k}\right) .
$$

Now use the relations $f_{1}, f_{3}$ and $f_{5}$ of (0.3.2) to rewrite this expression in terms of the basis $\left\{x_{i}^{2}, x_{0} x_{j}, x_{j} x_{0}: 0 \leq i \leq 3,1 \leq j \leq 3\right\}$ of $S_{2}$. Then, the coefficient of $x_{k}^{2}$ is still $d_{k k}$, whence $d_{k k}=0$. The coefficients of $x_{0} x_{1}$ and $x_{1} x_{0}$ are, respectively, $d_{01}+\left(1 / \alpha_{1}\right) d_{23}$ and $d_{01}-\left(1 / \alpha_{1}\right) d_{23}$. Thus, $d_{01}=0=$ $d_{23}$. Continuing in this manner, one proves that each $d_{k l}$ is zero, contradicting the fact that $A$ is nonsingular.

(ii) Suppose that there is an isomorphism $\psi: S_{\infty} \rightarrow S_{d, 2}$. Repeat the above argument, using the same notation, but with $S$ replaced by $S_{d, 2}$. Then everything up to and including (2.15.1) remains unchanged. In this case, $\left(S_{d, 2}\right)_{2}$ has basis $\left\{x_{1}^{2}, x_{2}^{2}, x_{3}^{2}, x_{2} x_{3}, x_{0} x_{j}, x_{j} x_{0}: 1 \leq j \leq 3\right\}$. Thus, the relations $f_{1}, f_{3}$ and $f_{5}$ can still be used to imply that $\bar{d}_{k l}=0$ if $k \neq l$. However, as $x_{0}^{2}=c_{1} x_{1}^{2}+c_{2} x_{2}^{2}+c_{3} x_{3}^{2}$, for some $c_{j} \in k$, we can only conclude that $d_{j j}=c_{j} d_{00}$ for each $j$. This implies that, for each choice of basis $\left\{r_{i}\right\}$ of $\left(S_{d, i}\right)_{1}$ there is, up to a scalar multiple, only one sum of squares $\sum \lambda_{i} r_{i}^{2}$ that can be zero. Clearly, this is sufficient to show that $S_{d, 2} \not S_{\infty}\left(\gamma_{1}, \gamma_{2}, \gamma_{3}\right)$, for any $\gamma_{i}$. The proof for $S_{d, 1}$ is similar.

2.16. A natural question raised by Lemma 2.15 , and one we now address, is to determine the isomorphism classes within the three classes, $\left\{S\left(\alpha_{1}, \alpha_{2}, \alpha_{3}\right)\right\}$, $\left\{S_{d, i}\left(\alpha_{1}, \alpha_{2}, \alpha_{3}\right)\right\}$, and $\left\{S_{\infty}\left(\alpha_{1}, \alpha_{2}, \alpha_{3}\right)\right\}$. To this end, we recall the notation of (1.1) and (1.2). In particular, if $R_{Y} \in \mathscr{Y}$, then we will usually write $R_{Y}=R_{Y}\left(\alpha_{1}, \alpha_{2}, \alpha_{3}\right)$, for the appropriate choice of $\alpha_{j}$ to emphasize the dependence of $R_{Y}$ on those scalars. If $d=\left(1, d_{2}\right) \in \mathbb{P}_{k}^{1}$, then $\left\{\alpha_{1}, \alpha_{2}, \alpha_{3}, d\right\}$ is called in general position if $\alpha_{1}, \alpha_{2}$ and $d_{2}$ are algebraically independent over the prime subfield of $k$ while $\alpha_{3}$ is determined by $(0.3 .1)$.

Lemma. (i) If $R_{Y}=S_{d, i}\left(\alpha_{1}, \alpha_{2}, \alpha_{3}\right) \in \mathscr{Y}$, then the associated variety $\Gamma_{Y} \subset$ $\mathbb{P}^{3} \times \mathbb{P}^{3}$ is the union $\Gamma_{Y}=\Gamma_{1}\left(\alpha_{1}, \alpha_{2}, \alpha_{3}\right) \cup F_{Y}$ for a finite set of points $F_{Y}$.

(ii) Assume that $\left\{\alpha_{1}, \alpha_{2}, \alpha_{3}, d=\left(1, d_{2}\right)\right\}$ is in general position, and that $R_{Y}=S_{d, 1}$. Let $\pi_{1}: \mathbb{P}^{3} \times \mathbb{P}^{3} \rightarrow \mathbb{P}^{3}$ be the projection onto the first coordinate. Then $\left.\pi_{1}\right|_{\Gamma_{Y}}$ is injective. Moreover, $G_{Y}=\pi_{1}\left(F_{Y}\right)$ consists of the four points $\left\{\left(x_{0}, x_{1}, x_{2}, x_{3}\right)=(0,0,1, \pm s),(1, \pm t, 0,0)\right.$. The values of $s$ and $t$ are obtained by ensuring that these points are zeros of $f_{2}$ and $\Omega_{1}+d_{2} \Omega_{2}$.

(iii) If $R_{Y}=S_{\infty}\left(\alpha_{1}, \alpha_{2}, \alpha_{3}\right) \in \mathscr{Y}$, then the associated variety $\Gamma_{Y} \subset \mathbb{P}^{3} \times \mathbb{P}^{3}$ is the union $\Gamma_{Y}=\Gamma_{1}\left(\alpha_{1}, \alpha_{2}, \alpha_{3}\right) \cup F_{Y}$ where $F_{Y}$ is now the union of two lines. 
In the notation of (ii), $\left.\pi_{1}\right|_{\Gamma_{Y}}$ is still injective and $G_{Y}$ consists of the lines

$$
x_{0}= \pm u v x_{3}, \quad x_{1}= \pm u x_{2} / v .
$$

Here $u=\sqrt{-\left(\alpha_{3}+\alpha_{2}\right) /\left(\alpha_{2} \alpha_{3}+1\right)}$ and $v=\sqrt{b}$ (thus one must take the same square roots in the two parts of (2.16.1)).

Proof. As with the corresponding proof for $S\left(\alpha_{1}, \alpha_{2}, \alpha_{3}\right)$ in [SS], this is a tedious exercise and is omitted.

Remark. Part (iii) of the lemma implies that there is a second geometric ring associated to $S_{\infty}\left(\alpha_{1}, \alpha_{2}, \alpha_{3}\right)$, to wit the one corresponding to the variety $F_{Y}$.

2.17 Lemma. Let $R_{Y}=R_{Y}\left(\alpha_{1}, \alpha_{2}, \alpha_{3}\right) \in \mathscr{Y}$. Then $\Gamma_{1}\left(\alpha_{1}, \alpha_{2}, \alpha_{3}\right)$ is an invariant of $R_{Y}$ as an element of $\mathscr{Y}$. Moreover, so are the elliptic curve $E=$ $E\left(\alpha_{1}, \alpha_{2}, \alpha_{3}\right)$ and the geometric ring $B=B\left(\alpha_{1}, \alpha_{2}, \alpha_{3}\right)$.

Proof. Suppose that $\phi: R_{Z} \rightarrow R_{Y}$ is an isomorphism and set $s_{j}=\phi\left(x_{j}\right)$, for $0 \leq j \leq 3$. As in the proof of Lemma 2.15, we may write $s_{j}=r_{j}+h_{j}$, where $r_{j} \in\left(R_{Y}\right)_{1}$ and $h_{j} \in \bigoplus_{m \geq 2}\left(R_{Y}\right)_{m}$. Moreover, the quadratic relations satisfied by the $s_{j}$ (that is, $Z$ ) are also satisfied by the $r_{j}$. Let $\left\{g_{k}=\sum_{i, j} g_{i j}^{k} x_{i} x_{j}\right\}$ be a basis for $Z$. If $r_{i}=\sum a_{i j} x_{j}$, then the relations $g_{k}^{\prime}=\sum_{i, j} g_{i j}^{k} a_{i u} a_{j v} x_{u} x_{v}=0$ hold in $R_{Y}$. Since the $r_{j}$ are linearly independent so are the $g_{k}^{\prime}$. Thus the $g_{k}^{\prime}$ form a basis for $Y$ and define $\Gamma_{Y}$. But, this implies that the automorphism $\phi_{1}: \mathbb{P}^{3} \times \mathbb{P}^{3}$ given by $x_{i j} \rightarrow \sum a_{i l} x_{l j}$, for $0 \leq i \leq 3$ and $j=1,2$, maps $\Gamma_{Z}$ to $\Gamma_{Y}$. Thus $\Gamma_{Y}$ is an invariant for $R_{Y}$. Since $\Gamma_{1}$ is isomorphic to an elliptic curve, Lemma 2.16 implies that $\Gamma_{1}\left(\alpha_{1}, \alpha_{2}, \alpha_{3}\right)$ is also an invariant and by projecting $\Gamma_{1}\left(\alpha_{1}, \alpha_{2}, \alpha_{3}\right)$ onto the first copy of $\mathbb{P}^{3}$, so is $E=E\left(\alpha_{1}, \alpha_{2}, \alpha_{3}\right)$.

Finally, consider the geometric ring $B$, which we will write as $B_{Y}=$ $B_{Y}\left(\alpha_{1}, \alpha_{2}, \alpha_{3}\right)=T(V) /\left\langle W_{Y}\right\rangle$ since we wish to consider it as a factor ring of $R_{Y}$. The proof of [SS, Lemma 3.3] shows that $W_{Y}$ is precisely the set of elements in $V \otimes V$ that are zero on $\Gamma_{1}\left(\alpha_{1}, \alpha_{2}, \alpha_{3}\right)$. Thus $\phi$ must map $W_{Z}$ to $W_{Y}$ and hence induce an isomorphism from $B_{Z}$ to $B_{Y}$.

2.18. As was remarked in the proof of Proposition 2.10, cyclically permuting the variables $\left\{x_{1}, x_{2}, x_{3}\right\}$ provides isomorphisms

$$
B\left(\alpha_{1}, \alpha_{2}, \alpha_{3}\right) \cong B\left(\alpha_{2}, \alpha_{3}, \alpha_{1}\right) \cong B\left(\alpha_{3}, \alpha_{1}, \alpha_{2}\right) .
$$

Similarly, the maps $\left(x_{0}, x_{1}, x_{2}, x_{3}\right) \rightarrow\left(x_{0}, x_{1},-x_{3}, x_{2}\right)$ and

$$
\left(x_{0}, x_{1}, x_{2}, x_{3}\right) \rightarrow\left(x_{1}, \frac{i}{\sqrt{\alpha_{2} \alpha_{3}}} x_{0}, \frac{i}{\sqrt{\alpha_{3}}} x_{2}, \frac{1}{\sqrt{\alpha_{2}}} x_{3}\right)
$$

both provide isomorphisms $B\left(\alpha_{1}, \alpha_{2}, \alpha_{3}\right) \cong B\left(-\alpha_{1},-\alpha_{3},-\alpha_{2}\right)$. These same permutations of the variables also induce isomorphisms between the appropriate $S\left(\alpha_{1}, \alpha_{2}, \alpha_{3}\right)$ or, more generally, between the appropriate $R_{Y}$. In each case, the group of isomorphisms induced by those described above will be called the permutation isomorphisms. A useful observation is that, as permutations of the variables $x_{j}$, one obtains the full permutation group on four letters. It is not difficult to prove that the permutation isomorphisms are, essentially, the only isomorphisms between the $B\left(\alpha_{1}, \alpha_{2}, \alpha_{3}\right)$ : 
Lemma. Suppose that $B\left(\alpha_{1}, \alpha_{2}, \alpha_{3}\right) \stackrel{\phi}{\cong} B\left(\beta_{1}, \beta_{2}, \beta_{3}\right)$ for some $\alpha_{i}$ and $\beta_{j}$ that satisfy (0.3.1) and (0.3.3). Then $B\left(\alpha_{1}, \alpha_{2}, \alpha_{3}\right)$ and $B\left(\beta_{1}, \beta_{2}, \beta_{3}\right)$ are also isomorphic via a permutation isomorphism.

Proof. Set $B=B\left(\alpha_{1}, \alpha_{2}, \alpha_{3}\right)$. Once again we follow the proof of Lemma 2.15 and write $\phi\left(x_{j}\right)=s_{j}=r_{j}+h_{j}$, where $r_{j} \in B_{1}$ and $h_{j} \in \bigoplus_{m>2} B_{m}$. Write $r_{j}=\sum a_{j k} x_{k}$ and $A=\left(a_{j k}\right)$. In this case, the $r_{j}$ satisfy two linearly independent equations involving sums of squares: $-r_{0}^{2}+r_{1}^{2}+r_{2}^{2}+r_{3}^{2}=0$ and

$$
r_{1}+c_{2} r_{2}^{2}+c_{3} r_{3}^{2}=0, \quad \text { where } c_{2}=\frac{1+\beta_{1}}{1-\beta_{2}} \text { and } c_{3}=\frac{1-\beta_{1}}{1+\beta_{3}} \text {. }
$$

It is important to note that $(0.3 .1)$ and (0.3.3) ensure that $0,1, c_{2}, c_{3}$ are always distinct. Define the diagonal matrices $\Lambda_{1}=\operatorname{diag}(-1,1,1,1)$ and $\Lambda_{2}=\operatorname{diag}\left(0,1, c_{2}, c_{3}\right)$. Then part (ii) of the proof of Lemma 2.15 implies that $D_{1}=A^{T} \Lambda_{1} A$ and $D_{2}=A^{T} \Lambda_{2} A$ are diagonal. Since $\Lambda_{1}^{-1} \Lambda_{2}=\Lambda_{2}$, this implies that

$$
A^{-1} \Lambda_{2} A=D_{1}^{-1} D_{2}=D_{3}=\operatorname{diag}\left(\delta_{0}, \delta_{1}, \delta_{2}, \delta_{3}\right)
$$

for some $\delta_{j} \in k$. Since $D_{3}$ is diagonal, this implies that the columns of $A$ are eigenvectors for $\Lambda_{2}$. Since the corresponding eigenvalues are distinct, this forces $A$ to have just one nonzero entry in each row and column. In other words, $r_{j}=\mu_{j} x_{p(j)}$ for some nonzero scalars $\mu_{j}$ and permutation $p$. By applying the permutation isomorphisms, and adjusting the $\alpha_{j}$ and $\mu_{l}$ accordingly, we may assume that $x_{p(j)}=x_{j}$ for $0 \leq j \leq 3$. Since we are working with graded rings, we may multiply each $r_{j}$ by the same scalar and also assume that $\mu_{0}=1$. It is now routine to use the relations $f_{i}$ of $(0.3 .2)$ to prove that $\mu_{j}=1$ for each $j$ and hence that $\beta_{k}=\alpha_{k}$ for each $k$.

2.19. By combining the last two lemmas one obtains:

Proposition. Given $R_{Y} \in \mathscr{Y}$, then there are at most finitely many $R_{Z} \in \mathscr{Y}$ with $R_{Z} \cong R_{Y}$.

Obviously, the proposition can be made much more precise and we leave the details to the interested reader.

2.20. Recall that, if $S=S\left(\alpha_{1}, \alpha_{2}, \alpha_{3}\right)$, then $B\left(\alpha_{1}, \alpha_{2}, \alpha_{3}\right) \cong S / \Omega_{1} S+\Omega_{2} S$ where the $\Omega_{j}$ are central in $S$. Analogously, if $d=\left(d_{1}, d_{2}\right) \neq(1,0)$ then $B \cong S_{d, 1} / \Omega_{1} S_{d, 1}+f_{1} S_{d, 1}$. In this case, direct computations show that $\Omega_{1}$ is still central in $S_{d, 1}$ but $f_{1}$ is only normal: in the notation of (2.3),

$$
\left(x_{0}, x_{1}, x_{2}, x_{3}\right)^{f_{1}}=\left(-x_{0},-x_{1}, x_{2}, x_{3}\right) .
$$

Similarly, if $d \neq(1,0)$, then $B \cong S_{d, 2} / \Omega_{1} S_{d, 2}+f_{2} S_{d, 2}$, where $\Omega_{1}$ is central and

$$
\left(x_{0}, x_{1}, x_{2}, x_{3}\right)^{f_{2}}=\left(x_{0}, x_{1},-x_{2},-x_{3}\right) .
$$

Likewise, $B \cong S_{\infty} / f_{1} S_{\infty}+f_{2} S_{\infty}$ where $f_{1}$ and $f_{2}$ satisfy (2.20.1) and (2.20.2).

Assume, now, that the $\alpha_{j}$ are in general position. Then one can prove that $\Omega_{1}$ is the only central element in $\left(S_{d, j}\right)_{2}$ while $\left(S_{\infty}\right)_{2}$ contains no central elements. 


\section{The Algebra $B\left(\alpha_{1}, \alpha_{2}, \alpha_{3}\right)$ Is Koszul}

3.1. Let $B=B\left(\alpha_{1}, \alpha_{2}, \alpha_{3}\right)$ be the geometric ring as defined in (0.4) or (1.2). In this section we will substantiate a claim made in (2.3), by giving an alternative proof that $\operatorname{dim}_{k}\left(B_{n}\right)=4 n$ for all $n \geq 1$. The idea is as follows. First, we prove that $B$ is a Koszul algebra (see Theorem 3.9). It then follows from [Lo, Theorem 1.2] that $H_{B^{!}}(t)=1 / H_{B}(-t)$. Since [SS, Theorem 3.4(ii)] shows that $H_{B}(t)=(1+t)^{2} /(1-t)^{2}$, this implies that $H_{B^{!}}(t)$ also equals $(1+t)^{2} /(1-t)^{2}$, as required. The proof that $B$ is Koszul uses the techniques from [Mu].

We will actually prove the result in slightly more generality than that required for this paper, since the extra generality requires no extra effort and may be useful elsewhere. Thus, in this section we will assume that $\mathscr{L}$ is a very ample, invertible sheaf over the elliptic curve $E$ such that $r=\operatorname{deg} \mathscr{L} \geq 4$. However, as before, $\tau$ will denote an automorphism of $E$ and the construction of $\mathscr{L}_{n}$ and $B_{n}=H^{0}\left(E, \mathscr{L}_{n}\right)$ and $B=\bigoplus_{n>0} B_{n}$ is the same as outlined in 1.2. This is the same generality in which the results of [SS, §3] were proved and so, by [SS, Theorem 3.4], $B=T(V) /\langle W\rangle$, where $V=H^{0}(E, \mathscr{L})=B_{1}$ and $W$ is an $\left(r^{2}-2 r\right)$-dimensional subspace of $V \otimes V$.

3.2. The definition of a Koszul algebra given in (0.3) is not convenient for our purposes and so we begin with an equivalent definition. This is taken from [Lo], and the reader is referred to that paper for the proofs and other details. Let $\phi: V \otimes V \rightarrow B_{2}$ be the multiplication map; thus $W=\operatorname{ker}(\phi)$. Inductively, define graded subspaces $K_{n} \subseteq K_{n-1} \otimes V \subseteq V^{\otimes n}$ by $K_{0}=k, K_{1}=V$ and, for $n \geq 1$,

$$
K_{n+1}=\operatorname{Ker}\left(K_{n} \otimes V \stackrel{i_{n} \otimes 1}{\longrightarrow} K_{n-1} \otimes V \otimes V \stackrel{1 \otimes \phi}{\longrightarrow} K_{n-1} \otimes B_{2}\right),
$$

where $i_{n}: K_{n} \subseteq K_{n-1} \otimes V$ is the given inclusion. Equivalently, for $n \geq 1$,

$$
K_{n+1}=\bigcap_{i=0}^{n-1} V^{\otimes i} \otimes \operatorname{ker}(\phi) \otimes V^{\otimes(n-i-1)}
$$

If $\phi_{t}: V \otimes B_{t} \rightarrow B_{t+1}$ denotes the multiplication map, then there is a natural map

$$
d_{n}^{t}: K_{n} \otimes B_{t} \stackrel{i_{n} \otimes 1}{\longrightarrow} K_{n-1} \otimes V \otimes B_{t} \stackrel{1 \otimes \phi_{t}}{\longrightarrow} K_{n-1} \otimes B_{t+1},
$$

where $d_{0}^{t}\left(K_{0} \otimes B_{t}\right)$ is defined to be zero. It is easy to check that $d_{n-1}^{t+1} d_{n}^{t}=0$ and hence that this defines a complex $\left(U^{\bullet}, d\right)$, where $U^{\bullet}=\bigoplus_{n>1} K_{n} \otimes B$. The significance of this complex is the following result (see [Lo, Theorem 1.2, Proposition 1.2 and Theorem 1.1]).

Proposition. (i) The complex $\left(U^{\bullet}, d\right)$ is acyclic if and only if $B$ is Koszul.

(ii) Let $\left[\operatorname{Ext}_{B}^{1}(k, k)\right]$ denote the subring of the $\operatorname{Ext}$ algebra $\operatorname{Ext}_{B}^{*}(k, k)$ generated by $\operatorname{Ext}_{B}^{1}(k, k)$. Then $\prod_{n \geq 0} K_{n} \cong\left[\operatorname{Ext}_{B}^{1}(k, k)\right] \cong B^{!}$as bigraded vector spaces.

3.3. In order to apply [Mu], we need to reinterpret the $K_{n}$ and (3.2.1) in terms of (homomorphisms between) global sections of locally free $\mathscr{O}_{E}$-modules. If $\mathscr{X}$ and $\mathscr{Y}$ are locally free $\mathscr{O}_{E}$-modules, we will write $H^{j}(\mathscr{X})=H^{j}(E, \mathscr{X})$ and let

$$
\mu(\mathscr{X}, \mathscr{Y}): H^{0}(\mathscr{X}) \otimes H^{0}(\mathscr{Y}) \longrightarrow H^{0}(\mathscr{X} \otimes \mathscr{Y})
$$


denote the natural map obtained by taking global sections of $\mathscr{X} \otimes \mathscr{Y}$. Following [Mu], write

$$
R(\mathscr{X}, \mathscr{Y})=\operatorname{Ker}(\mu(\mathscr{X}, \mathscr{Y})) \text { and } S(\mathscr{X}, \mathscr{Y})=\operatorname{Coker}(\mu(\mathscr{X}, \mathscr{Y})) \text {. }
$$

In particular, $K_{2}=\operatorname{ker}(\phi)=R\left(\mathscr{L}, \mathscr{L}^{\tau}\right)$. (The automorphism $\tau$ appears in this description since, in the present notation, multiplication in $B$ is defined by $u \cdot v=\mu\left(\mathscr{L}_{n}, \mathscr{L}_{m}^{\tau^{n}}\right)\left(u \otimes v^{\tau^{n}}\right)$, for $u \in B_{n}$ and $\left.v \in B_{m}.\right)$

Next, we use an idea from [ATV1]; see in particular [ATV1, Proposition 1.17]. If $\mathscr{X}$ is a locally free $\mathscr{O}_{E}$-module that is generated by its global sections, write $\mathscr{O}^{\mathscr{X}}$ for $\mathscr{O}_{E}^{H^{0}(\mathscr{X})}$ and let $P_{\mathscr{Z}}$ denote the kernel of the natural surjection $\mathscr{O}^{\mathscr{X}} \rightarrow \mathscr{X}$. The significance of this occurs when $H^{1}(\mathscr{Y})=0$. For, in this case, take global sections of the exact sequence

$$
P_{\mathscr{X}} \otimes \mathscr{Y} \longrightarrow \mathscr{O}^{\mathscr{X}} \otimes \mathscr{Y} \longrightarrow \mathscr{X} \otimes \mathscr{Y}
$$

and use the fact that $H^{1}\left(\mathscr{O}^{\mathscr{Z}} \otimes \mathscr{Y}\right)=H^{0}(\mathscr{X}) \otimes H^{1}(\mathscr{Y})=0$. Then one then obtains the exact sequence

(3.3.1)

$0 \longrightarrow H^{0}\left(P_{\mathscr{X}} \otimes \mathscr{Y}\right) \longrightarrow H^{0}(\mathscr{X}) \otimes H^{0}(\mathscr{Y}) \longrightarrow H^{0}(\mathscr{X} \otimes \mathscr{Y}) \longrightarrow H^{1}\left(P_{\mathscr{Z}} \otimes \mathscr{Y}\right) \longrightarrow 0$.

Thus, one has the natural identifications $H^{0}\left(P_{\mathscr{X}} \otimes \mathscr{Y}\right)=R(\mathscr{X}, \mathscr{Y})$ and $H^{1}\left(P_{\mathscr{X}} \otimes \mathscr{Y}\right)=S(\mathscr{X}, \mathscr{Y})$. In particular, $K_{2} \cong H^{0}\left(P_{\mathscr{L}} \otimes \mathscr{L}^{\tau}\right)$. The main aim of this section is to find similar identifications for the other groups $K_{s}$, since this will allow us to give an easy proof that $\left(U^{\bullet}, d\right)$ is exact.

Before we can do this, however, we need to show that various $\mathscr{O}_{E}$-modules are generated by their sections. Let $\mathscr{X}$ be a locally free $\mathscr{O}_{E}$-module of rank $t$ and $\left\{\mathscr{M}_{j}: 1 \leq j \leq t\right\}$ be invertible $\mathscr{O}_{E}$-modules. Following [At], $\left(\mathscr{M}_{1}, \mathscr{M}_{2}, \ldots, \mathscr{M}_{t}\right)$ is called a splitting of $\mathscr{X}$ if there exists a series $0=\mathscr{X}_{0} \subseteq \mathscr{X}_{1} \subset \cdots \subset \mathscr{X}_{t}=\mathscr{X}$ of $\mathscr{O}_{E}$-submodules of $\mathscr{X}$ such that $\mathscr{M}_{j}=\mathscr{X}_{j} / \mathscr{X}_{j-1}$, for $1 \leq j \leq t$. We will call this series $\left\{\mathscr{X}_{j}\right\}$ the splitting series of $\mathscr{X}$ corresponding to the splitting $\left(\mathscr{M}_{1}, \mathscr{M}_{2}, \ldots, \mathscr{M}_{t}\right)$.

Proposition. Let $\mathscr{K}$ be an invertible $\mathscr{O}_{E}$-module with $\operatorname{deg} \mathscr{K}=r \geq 4$ and $\mathscr{X}$ a locally free $\mathscr{O}_{E}$-module satisfying:

$\mathscr{X}$ is generated by its sections and $S(\mathscr{X}, \mathscr{M})=0$ for all but finitely many invertible $\mathscr{O}_{E}$-modules $\mathscr{M}$ that satisfy $\operatorname{deg} \mathscr{M}=2$.

Then $P_{\mathscr{X}} \otimes \mathscr{K}$ satisfies (3.3.2). Indeed, $P_{\mathscr{Z}} \otimes \mathscr{K}$ has a splitting $\left(\mathscr{N}_{1}, \ldots, \mathscr{N}_{S}\right)$ such that $\operatorname{deg} \mathscr{N}_{j} \geq 2$ for all $j$.

Remark. By [Ha, Example 1.3.7, p. 297], there exist infinitely many invertible $\mathscr{O}_{E}$-modules $\mathscr{M}$ with $\operatorname{deg} \mathscr{M}=2$.

Proof. Pick an invertible $\mathscr{O}_{E}$-module $\mathscr{M}$ such that $S(\mathscr{X}, \mathscr{M})=0$ and $\operatorname{deg} \mathscr{M}$ $=2$. Since $H^{1}(\mathscr{M})=0$, the comments after (3.3.1) imply that $H^{1}\left(P_{\mathscr{L}} \otimes \mathscr{M}\right)=$ 0 . Now suppose that $P_{\mathscr{Z}} \otimes \mathscr{K}=\mathscr{Y} \oplus \mathscr{Z}$, with $\mathscr{Y}$ irreducible. Clearly, in order to prove that $P_{\mathscr{Z}} \otimes \mathscr{K}$ has a splitting $\left(\mathscr{N}_{1}, \ldots, \mathscr{N}_{s}\right)$ such that $\operatorname{deg} \mathscr{N}_{j} \geq 2$ for all $j$, it suffices to prove that same fact for $\mathscr{Y}$. Set $\mathscr{G}=\mathscr{M}^{-1} \otimes \mathscr{K}$ and note that $\operatorname{deg} \mathscr{G}=(r-2) \geq 2$. Write $\widetilde{\mathscr{Y}}=\mathscr{Y} \otimes \mathscr{G}^{-1}$. Since $\widetilde{\mathscr{Y}}$ is a summand of $P_{\mathscr{X}} \otimes \mathscr{M}$, certainly $H^{1}(\widetilde{\mathscr{Y}})=0$. Thus, by the Riemann-Roch theorem, deg $\widetilde{\mathscr{Y}} \geq 0$. If $\operatorname{deg} \widetilde{\mathscr{Y}}>0$, then [At, Lemma $6^{\prime}$ and p. 415] implies that $\widetilde{\mathscr{Y}}$ has a splitting 
$\left(\mathscr{M}_{1}, \ldots, \mathscr{M}_{t}\right)$ such that $\mathscr{M}_{j} \geq \mathscr{M}_{1} \geq \mathscr{O}_{E}$ and hence $\operatorname{deg} \mathscr{M}_{j} \geq \operatorname{deg} \mathscr{M}_{1} \geq 0$ for all $j$. Hence $\mathscr{Y}$ has a splitting $\left(\mathscr{N}_{1}, \ldots, \mathscr{N}_{t}\right)$, where

$$
\operatorname{deg} \mathscr{N}_{j}=\operatorname{deg} \mathscr{M}_{j} \otimes \mathscr{G} \geq \operatorname{deg} \mathscr{G}=r-2 \geq 2,
$$

as required. Thus we may assume that $\operatorname{deg} \widetilde{\mathscr{Y}}=0$. Pick an invertible sheaf $\mathscr{C}$ with $\operatorname{deg} \mathscr{C}=1$. Thus, by the additivity of degree on short exact sequences,

$$
\operatorname{deg}(\widetilde{\mathscr{Y}} \otimes \mathscr{C})=\operatorname{deg} \widetilde{\mathscr{Y}}+(\operatorname{deg} \mathscr{C}) \operatorname{rank} \widetilde{\mathscr{Y}}=\operatorname{rank} \widetilde{\mathscr{Y}}
$$

In this case, [At, Lemma 11] implies that $\widetilde{\mathscr{Y}} \otimes \mathscr{C}$ has a splitting $(\mathscr{Q}, \ldots, \mathscr{Q})$, where $\operatorname{deg} \mathscr{Q}=1$. Hence $\mathscr{Y}$ has a splitting $\left(\mathscr{M}_{1}, \ldots, \mathscr{M}_{1}\right)$, where $\operatorname{deg} \mathscr{M}_{1}=$ $\operatorname{deg} \mathscr{Q} \otimes \mathscr{C}^{-1} \otimes \mathscr{G}=\operatorname{deg} \mathscr{G}=2$. Thus, we have proved that $P_{\mathscr{Z}} \otimes \mathscr{K}$ has a splitting $\left(\mathscr{N}_{1}, \ldots, \mathscr{N}_{s}\right)$ such that $\operatorname{deg} \mathscr{N}_{j} \geq 2$ for all $j$.

It now follows easily that $P_{\mathscr{X}} \otimes \mathscr{K}$ satisfies (3.3.2). To see this, let $\left\{\mathscr{H}_{j}\right\}$ be the splitting series of $P_{\mathscr{Z}} \otimes \mathscr{K}$ corresponding to the splitting $\left(\mathscr{N}_{1}, \ldots, \mathscr{N}_{s}\right)$. Thus, for each $j$, there exists a short exact sequence

$$
0 \longrightarrow \mathscr{N}_{j} \longrightarrow P_{\mathscr{Z}} \otimes \mathscr{K} / \mathscr{H}_{j-1} \longrightarrow P_{\mathscr{Z}} \otimes \mathscr{K} / \mathscr{H}_{j} \longrightarrow 0
$$

with $\mathscr{H}_{1}=\mathscr{N}_{1}$ and $P_{\mathscr{Z}} \otimes \mathscr{K} / \mathscr{H}_{s-1}=\mathscr{N}_{s}$. By [Ha, Corollary 3.2, p. 308], each $\mathscr{N}_{j}$ is generated by its sections. Thus the obvious induction, using (3.3.3) and [At, Lemma 1], implies that $P_{\mathscr{X}} \otimes \mathscr{K}$ is also generated by its sections and that $H^{1}\left(P_{\mathscr{Z}} \otimes \mathscr{K}\right)=0$. Next, let $\mathscr{M}$ be an invertible $\mathscr{O}_{E}$-module with $\operatorname{deg} \mathscr{M}=2$. Note that $\operatorname{deg} \mathscr{N}_{j} \otimes \mathscr{M}^{-1} \geq 0$. Thus, if $\mathscr{M} ¥ \mathscr{N}_{j}$ then [Ha, Lemma 1.2 , p.295] implies that $H^{1}\left(\mathscr{N}_{j} \otimes \mathscr{M}^{-1}\right)=0$. Hence [Mu, Theorem 2] implies that $S\left(\mathscr{N}_{j}, \mathscr{M}\right)=0$. So, assume that $\mathscr{M} \not \mathscr{N}_{j}$ for any $1 \leq j \leq s$. Since $H^{1}\left(\mathscr{N}_{j}\right)=0$ for each $j,(3.3 .3)$ and [Mu, 6-lemma] imply that there exist exact sequences

$$
S\left(\mathscr{N}_{j}, \mathscr{M}\right) \longrightarrow S\left(P_{\mathscr{Z}} \otimes \mathscr{K} / H_{j-1}, \mathscr{M}\right) \longrightarrow S\left(P_{\mathscr{Z}} \otimes \mathscr{K} / \mathscr{H}_{j}, \mathscr{M}\right) .
$$

Thus, by the obvious induction, one obtains that $S\left(P_{\mathscr{Z}} \otimes \mathscr{K}, \mathscr{M}\right)=0$. This completes the proof of the proposition.

3.4. The significance of Proposition 3.3 is that it allows us to make the following definition:

Corollary. Set $J_{2}=\mathscr{L}$ and, for $n \geq 2$, define $J_{n+1}=P_{J_{n}} \otimes \mathscr{L}^{\tau^{n-1}}$. By Proposition 3.3, $J_{n}$ is generated by its global sections and so $J_{n+1}$ is defined.

3.5 Corollary. Let $\mathscr{K}$ be an invertible $\mathscr{O}_{E}$-module with $\operatorname{deg} \mathscr{K} \geq 3$. Then $S\left(J_{s}, \mathscr{K}\right)=0$ for all $s \geq 2$.

Proof. By Proposition 3.3, there exists a splitting $\left(\mathscr{N}_{1}, \ldots, \mathscr{N}_{t}\right)$ of $J_{s}$, with corresponding splitting series $\left\{\mathscr{H}_{j}\right\}$, for which $\operatorname{deg} \mathscr{N}_{j} \geq 2$ for each $j$. As in (3.3.3), consider the exact sequences

$$
0 \longrightarrow \mathscr{N}_{j} \longrightarrow J_{s} / \mathscr{H}_{j-1} \longrightarrow J_{s} / H_{j} \longrightarrow 0 \text {. }
$$

Since $\operatorname{deg} \mathscr{K} \geq 3$ and $\operatorname{deg} \mathscr{N}_{j} \geq 2$, [Mu, Theorem 6] implies that $S\left(\mathscr{N}_{j}, \mathscr{K}\right)=$ 0 for each $j$. But, $H^{1}\left(\mathcal{N}_{j}\right)=0$ for each $j$. Thus [Mu, 6-lemma] implies that, for each $j$, there exists an exact sequence

$$
S\left(N_{j}, \mathscr{K}\right) \longrightarrow S\left(J_{s} / \mathscr{K}_{j-1}, \mathscr{K}\right) \longrightarrow S\left(J_{s} / \mathscr{K}_{j}, \mathscr{K}\right) .
$$

Thus, once again, the obvious induction implies that $S\left(J_{s}, \mathscr{K}\right)=0$. 
3.6 Lemma. Let $\mathscr{X}, \mathscr{Y}$, and $\mathscr{Z}$ be locally free $\mathscr{O}_{E}$-modules such that $\mathscr{X}$ and $\mathscr{Z}$ are generated by their sections. Then there are natural isomorphisms

$$
\begin{aligned}
H^{0}\left(P_{\mathscr{X}} \otimes \mathscr{Y} \otimes P_{\mathscr{X}}\right) & \cong R\left(P_{\mathscr{Z}} \otimes \mathscr{Y}, \mathscr{Z}\right) \\
& \cong \operatorname{Ker}\left(R(\mathscr{X}, \mathscr{Y}) \otimes H^{0}(\mathscr{Z}) \rightarrow R(\mathscr{X}, \mathscr{Y} \otimes \mathscr{Z})\right),
\end{aligned}
$$

and natural maps

$$
\begin{aligned}
S\left(P_{\mathscr{X}} \otimes \mathscr{Y}, \mathscr{Z}\right) & \cong \operatorname{Coker}\left(R(\mathscr{X}, \mathscr{Y}) \otimes H^{0}(\mathscr{Z}) \rightarrow R(\mathscr{X}, \mathscr{Y} \otimes \mathscr{Z})\right) \\
& \longrightarrow H^{1}\left(P_{\mathscr{Z}} \otimes \mathscr{Y} \otimes P_{\mathscr{X}}\right) .
\end{aligned}
$$

Proof. We have the following commutative diagram, where all the maps are the natural ones and each row and column is exact:

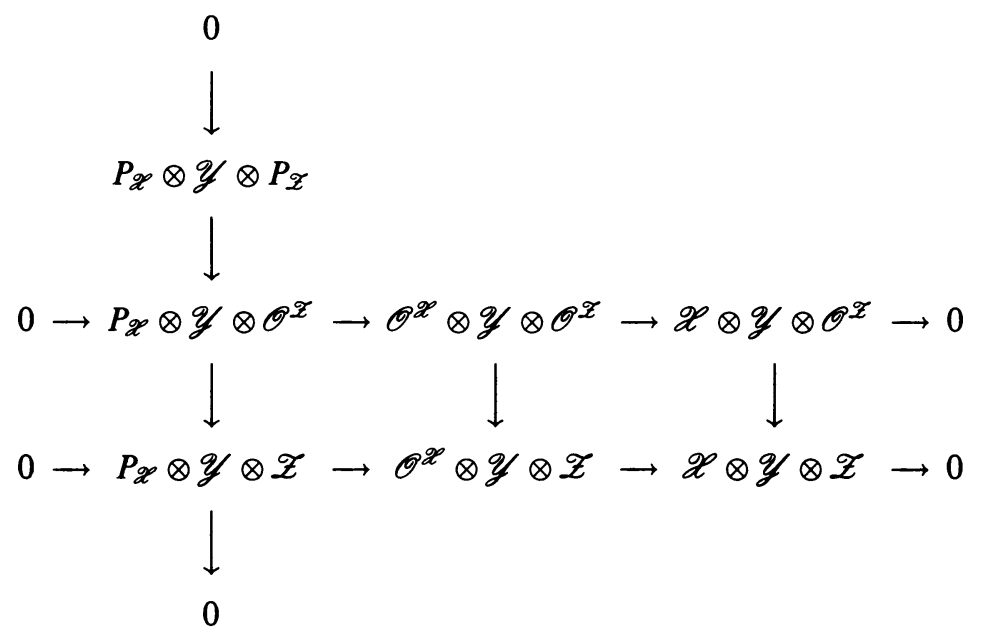

Taking global sections gives the following commutative diagram, again with exact rows and columns;

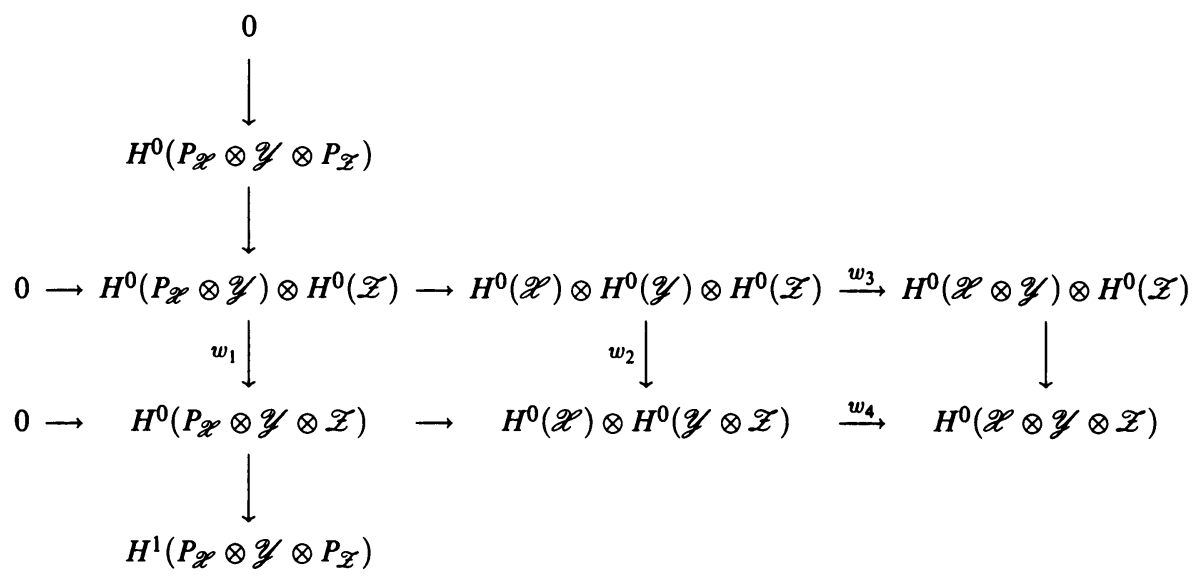

Here, $w_{3}$ and $w_{4}$ are the natural maps of global sections and so one obtains natural identifications:

$$
H^{0}\left(P_{\mathscr{L}} \otimes \mathscr{Y}\right) \otimes H^{0}(\mathscr{Z})=R(\mathscr{X}, \mathscr{Y}) \otimes H^{0}(\mathscr{Z})
$$


and

$$
H^{0}\left(P_{\mathscr{Z}} \otimes \mathscr{Y} \otimes \mathscr{Z}\right)=R(\mathscr{X}, \mathscr{Y} \otimes \mathscr{Z}) .
$$

Since $w_{2}$ is the natural map between the spaces of global sections, these identifications imply that $w_{1}$ is the natural map

$$
R(\mathscr{X}, \mathscr{Y}) \otimes H^{0}(\mathscr{Z}) \longrightarrow R(\mathscr{X}, \mathscr{Y} \otimes \mathscr{Z}) .
$$

Thus

$H^{0}\left(P_{\mathscr{X}} \otimes \mathscr{Y} \otimes P_{\mathscr{X}}\right)=\operatorname{Ker}\left(w_{1}\right)=\operatorname{Ker}\left(R(\mathscr{X}, \mathscr{Y}) \otimes H^{0}(\mathscr{Z}) \rightarrow R(\mathscr{X}, \mathscr{Y} \otimes \mathscr{Z})\right)$.

Now, consider the left-hand column of the diagram. Once again, the map $w_{1}$ is just the natural map of global sections, $\mu\left(P_{\mathscr{Z}} \otimes \mathscr{Y}, \mathscr{Z}\right)$. Thus one obtains natural identifications: $H^{0}\left(P_{\mathscr{Z}} \otimes \mathscr{Y} \otimes P_{\mathscr{Z}}\right)=R\left(P_{\mathscr{Z}} \otimes \mathscr{Y}, \mathscr{Z}\right)$ and

$$
S\left(P_{\mathscr{X}} \otimes \mathscr{Y}, \mathscr{Z}\right)=\operatorname{Coker}\left(w_{1}\right) \subseteq H^{1}\left(P_{\mathscr{Z}} \otimes \mathscr{Y} \otimes P_{\mathscr{Z}}\right),
$$

as required.

3.7 Corollary. For all $n \geq 2$ and $m \geq 1$, the natural map

$$
\psi: R\left(J_{n}, \mathscr{L}^{\tau^{n-1}}\right) \otimes H^{0}\left(\mathscr{L}_{m}^{\tau^{n}}\right) \longrightarrow R\left(J_{n}, \mathscr{L}_{m+1}^{\tau^{n-1}}\right)
$$

is surjective.

Proof. We will apply Lemma 3.6 with $\mathscr{X}=J_{n}, \mathscr{Y}=\mathscr{L}^{\tau^{n-1}}$, and $\mathscr{Z}=\mathscr{L}_{m}^{\tau^{n}}$. Note that the hypotheses of that lemma are satisfied, by Corollary 3.4. By Corollary 3.5

$$
0=S\left(J_{n+1}, \mathscr{L}_{m}^{\tau^{n}}\right)=S\left(P_{J_{n}} \otimes \mathscr{L}^{\tau^{n-1}}, \mathscr{L}_{m}^{\tau^{n}}\right)
$$

Thus, by the final assertion of Lemma 3.6, $\psi$ is indeed surjective.

3.8. We now return to the complex $\left(U^{\bullet}, d\right)$, as defined in 3.2 , and identify the various objects defined there in terms of global sections of $\mathscr{O}_{E}$-modules. Thus, for $n \geq 1$ and $s \geq 1$, let

$$
M_{n+1}^{s}=\operatorname{Ker}\left(K_{n} \otimes B_{s} \stackrel{d_{n}^{s}}{\longrightarrow} K_{n-1} \otimes B_{s+1}\right) .
$$

Recall that, by definition, $d_{0}^{s}=0$ and hence that $M_{1}^{s}=k \otimes B_{s}=B_{s}$.

Proposition. (i) For all $n \geq 2$ and $s \geq 1, M_{n}^{s}=R\left(J_{n}, \mathscr{L}_{s}^{\tau^{n-1}}\right)$. Similarly, $K_{0}=k, K_{1}=H^{0}(\mathscr{L})$, and $K_{n}=R\left(J_{n}, \mathscr{L}^{\tau^{n-1}}\right)$, for $n \geq 2$.

(ii) The complex $\left(U^{\bullet}, d\right)$ is acyclic.

Proof. (i) Since $K_{n}=M_{n}^{1}$, it suffices to prove the statement about the $M_{n}^{s}$, which we will do by induction on $n$. We will assume that $s \geq 1$ throughout the proof. By the comments in (3.3),

$$
\begin{aligned}
M_{2}^{s} & =\operatorname{Ker}\left(K_{1} \otimes B_{s} \rightarrow B_{s+1}\right)=\operatorname{Ker}\left(B \otimes B_{s} \rightarrow B_{s+1}\right) \\
& =R\left(\mathscr{L}, \mathscr{L}_{s}^{\tau}\right)=R\left(J_{2}, \mathscr{L}_{s}^{\tau}\right),
\end{aligned}
$$

as required. Now suppose that $n \geq 2$ and that $M_{m}^{s}=R\left(J_{m}, \mathscr{L}_{s}^{\tau^{m-1}}\right)$ for all $m \leq n$. As $\left(U^{\bullet}, d\right)$ is a complex,

$$
\operatorname{Im}\left(d_{n}^{s}\right) \subseteq \operatorname{Ker}\left(d_{n-1}^{s+1}\right)=M_{n}^{s+1}=R\left(J_{n}, \mathscr{L}_{s+1}^{\tau^{n-1}}\right)
$$


Similarly, $K_{n}=R\left(J_{n}, \mathscr{L}^{\tau^{n-1}}\right)$. Thus

$$
M_{n+1}^{s}=\operatorname{Ker}\left(R\left(J_{n}, \mathscr{L}^{\tau^{n-1}}\right) \otimes H^{0}\left(\mathscr{L}_{s}^{\tau^{n}}\right) \stackrel{d_{n}^{s}}{\longrightarrow} R\left(J_{n}, \mathscr{L}_{s+1}^{\tau^{n-1}}\right)\right) .
$$

By Corollary 3.4, and the fact that $r \geq 4$, both $\mathscr{X}=J_{n}$ and $\mathscr{Z}=\mathscr{L}_{s}{ }^{n}$ are generated by their sections. Thus the first assertion of Lemma 3.6, with $\mathscr{Y}=\mathscr{L}^{\tau^{n-1}}$, implies that

$$
M_{n+1}^{s}=R\left(P_{J_{n}} \otimes \mathscr{L}^{\tau^{n-1}}, \mathscr{L}_{s}^{\tau^{n}}\right)=R\left(J_{n+1}, \mathscr{L}_{s}^{\tau^{n}}\right) ;
$$

as required.

(ii) We begin with some special cases. By definition, $d_{n}^{0}: K_{n} \otimes B_{0} \rightarrow K_{n-1} \otimes$ $B_{1}$ is an injective map with image $K_{n}=M_{n}^{1}=\operatorname{Ker}\left(d_{n-1}^{1}\right)$, for any $n \geq 1$. Similarly, for any $s \geq 0$ the map $d_{0}^{s+1}$ is defined to be zero, while $d_{1}^{s}: K_{1} \otimes B_{s} \rightarrow$ $K_{0} \otimes B_{s+1}$ is just the multiplication map. This is surjective, by [Mu, Theorem 6]. Thus it remains to prove that $\operatorname{Im}\left(d_{n}^{s}\right)=\operatorname{Ker}\left(d_{n-1}^{s+1}\right)$ for all $n \geq 2$ and $s \geq 1$. But, by part(i), the homomorphism $d_{n}^{s}$, thought of as a map from $K_{n} \otimes B_{s}$ to $\operatorname{Ker}\left(d_{n-1}^{s+1}\right)$, is nothing more than the natural map

$$
R\left(J_{n}, \mathscr{L}^{\tau^{n-1}}\right) \otimes H^{0}\left(\mathscr{L}_{s}^{\tau^{n}}\right) \longrightarrow R\left(J_{n}, \mathscr{L}_{s+1}^{\tau^{n-1}}\right) .
$$

By Corollary 3.7 this is surjective.

3.9 Theorem. (i) If $B$ is defined as in (3.1), then $B$ is a Koszul algebra. Moreover, $B$ has Hilbert series

$$
H_{B}(t)=\sum_{n \geq 0} \operatorname{dim}_{k}\left(B_{n}\right)=\frac{1+(r-2) t+t^{2}}{(1-t)^{2}} .
$$

Similarly $B^{!}$has Hilbert series $H_{B^{\prime}}(t)=(1+t)^{2} /\left(1-(r-2) t+t^{2}\right)$.

(ii) In particular, if $B=B\left(\alpha_{1}, \alpha_{2}, \alpha_{3}\right)$ is the geometric algebra defined in the introduction, then $B$ is Koszul and

$$
H_{B}(t)=H_{B^{!}}(t)=(1+t)^{2} /(1-t)^{2} .
$$

Proof. That $B$ is Koszul follows from Propositions 3.2 and 3.8. By [SS, Theorem 3.4], $\operatorname{dim}_{k}\left(B_{n}\right)=r n$, from which the given value of $H_{B}(t)$ follows easily. Since [Lo, Theorem 1.2] implies that $H_{B}(t) H_{B^{\prime}}(-t)=1$ for a Koszul algebra $B$, the rest of part (i) follows. Part (ii) of the theorem is just the special case of (i) when $r=4$.

Remark. Part (ii) of the theorem gives the desired, alternative proof of Proposition 2.3(iii). Note that the theorem is independent of the automorphism $\tau$ of the elliptic curve $E$. Thus, if $C$ denotes the homogeneous coordinate ring of $E$ and the Ext algebra $\operatorname{Ext}_{B}^{*}(k, k)$ is given its usual bigraded structure (see [Lo]), then the theorem implies that (as $k$-vector spaces)

$$
\operatorname{Ext}_{B}^{p, q}(k, k)=\operatorname{Ext}_{C}^{p, q}(k, k)= \begin{cases}k^{(r p)}, & \text { if } p=q, \\ 0, & \text { if } p \neq q .\end{cases}
$$

If $\operatorname{deg} \mathscr{L}=3$, then the equality $\operatorname{Ext}_{B}^{p, q}(k, k)=\operatorname{Ext}_{C}^{p, q}(k, k)$ still holds, although the proof is harder. (This result is stated in [OF1, Lemma 2.2]. However, the proof as outlined in $[\mathrm{OF}]$ is incorrect, although Levasseur has found a correct argument.) One reason why the result should be more difficult when $\operatorname{deg} \mathscr{L}=3$ is that now the geometric ring $B$ is not a Koszul algebra, since one of its defining relations is cubic (see [ATV1]). 


\section{REFERENCES}

[AS] M. Artin and W. Schelter, Graded algebras of global dimension 3, Adv. Math. 66 (1987), 171-216.

[ATV1] M. Artin, J. Tate and M. Van den Bergh, Some algebras associated to automorphisms of curves, The Grothendieck Festschrift (P. Cartier, et al. eds.), Birkhäuser, 1990.

[ATV2] M. Artin, J. Tate and M. Van den Bergh, Modules over regular algebras of dimension 3, Invent. Math. 106 (1991), 335-388.

[At] M. F. Atiyah, Vector bundles over an elliptic curve, Proc. London. Math. Soc. (3) 7 (1957), 414-452.

[Ha] R. Hartshorne, Algebraic geometry, Springer-Verlag, 1977.

[Le] T. Levasseur, Some properties of non-commutative regular rings, Glasgow Math. J. 34 (1992), 277-300.

[Lo] C. Löfwall, On the subalgebra generated by the one-dimensional elements in the Yoneda Extalgebra, Algebra, Algebraic Topology and Their Interactions, (J.-E. Roos, ed.), Lecture Notes in Math., vol. 1183, Springer-Verlag, 1986.

[Ma] Yu. I. Manin, Quantum groups and non-commutative geometry, Publ. Centre Rech. Math., Univ. Montreal, 1988.

[Mu] D. Mumford, Varieties defined by quadratic relations, Questions on Algebraic Varieties, (E. Marchionne, ed.), C.I.M.E., III Ciclo, Varenna, 1969, Roma, 1970.

[OF1] A. V. Odesskii and B. L. Feigin, Sklyanin algebras associated with an elliptic curve, preprint 1989. (Russian)

[OF2] __ Elliptic Sklyanin algebras, Funktsional. Anal. i Prilozhen. 23 (1989), 45-54. (Russian)

[Pr] S. B. Priddy, Koszul resolutions, Trans. Amer. Math. Soc. 152 (1970), 39-60.

[Sk1] E. K. Sklyanin, Some algebraic structures connected to the Yang-Baxter equation, Functional Anal. Appl. 16 (1982), 27-34.

[Sk2] _ - Some algebraic structures connected to the Yang-Baxter equation. Representations of quantum algebras, Functional Anal. Appl. 17 (1983), 273-284.

[SS] S. P. Smith and J. T. Stafford, Regularity of the four dimensional Sklyanin algebra, Compositio Math. 83 (1992), 259-284.

[St] J. T. Stafford, Auslander-regular rings and maximal orders, J. London Math. Soc. (to appear).

Department of Mathematics, University of Michigan, AnN Arbor, Michigan 48109

E-mail address: jts@math.1sa.umich.edu 